\title{
Metabolic engineering for increased lipid accumulation in Yarrowia lipolytica - A
} Review

Wang, Jinpeng; Ledesma-Amaro, Rodrigo; Wei, Yongjun; Ji, Boyang; Ji, Xiao Jun

Published in:

Bioresource Technology

Link to article, DOI:

10.1016/j.biortech.2020.123707

Publication date:

2020

Document Version

Peer reviewed version

Link back to DTU Orbit

Citation (APA):

Wang, J., Ledesma-Amaro, R., Wei, Y., Ji, B., \& Ji, X. J. (2020). Metabolic engineering for increased lipid accumulation in Yarrowia lipolytica - A Review. Bioresource Technology, 313, [123707]. https://doi.org/10.1016/j.biortech.2020.123707

\section{General rights}

Copyright and moral rights for the publications made accessible in the public portal are retained by the authors and/or other copyright owners and it is a condition of accessing publications that users recognise and abide by the legal requirements associated with these rights.

- Users may download and print one copy of any publication from the public portal for the purpose of private study or research.

- You may not further distribute the material or use it for any profit-making activity or commercial gain

- You may freely distribute the URL identifying the publication in the public portal

If you believe that this document breaches copyright please contact us providing details, and we will remove access to the work immediately and investigate your claim 


\section{Journal Pre-proofs}

Review

Metabolic engineering for increased lipid accumulation in Yarrowia lipolytica

Jinpeng Wang, Rodrigo Ledesma-Amaro, Yongjun Wei, Boyang Ji, Xiao-Jun $\mathrm{Ji}$

PII:

S0960-8524(20)30979-2

DOI: https://doi.org/10.1016/j.biortech.2020.123707

Reference: BITE 123707

To appear in: $\quad$ Bioresource Technology

Received Date: $\quad 14$ May 2020

Revised Date: $\quad 15$ June 2020

Accepted Date: $\quad 16$ June 2020

Please cite this article as: Wang, J., Ledesma-Amaro, R., Wei, Y., Ji, B., Ji, X-J., Metabolic engineering for increased lipid accumulation in Yarrowia lipolytica, Bioresource Technology (2020), doi: https://doi.org/10.1016/ j.biortech.2020.123707

This is a PDF file of an article that has undergone enhancements after acceptance, such as the addition of a cover page and metadata, and formatting for readability, but it is not yet the definitive version of record. This version will undergo additional copyediting, typesetting and review before it is published in its final form, but we are providing this version to give early visibility of the article. Please note that, during the production process, errors may be discovered which could affect the content, and all legal disclaimers that apply to the journal pertain.

(C) 2020 Published by Elsevier Ltd. 
Metabolic engineering for increased lipid accumulation in Yarrowia lipolytica

Jinpeng Wang ${ }^{1}$, Rodrigo Ledesma-Amaro ${ }^{2}$, Yongjun $\mathrm{Wei}^{3}$, Boyang $\mathrm{Ji}^{4,5}$, Xiao-Jun $\mathrm{Ji}^{1 *}$

${ }^{1}$ College of Biotechnology and Pharmaceutical Engineering, Nanjing Tech University, No. 30 South Puzhu Road, Nanjing 211816, People's Republic of China

${ }^{2}$ Department of Bioengineering and Imperial College Centre for Synthetic Biology, Imperial College London, London, SW7 2AZ, U.K.

${ }^{3}$ School of Pharmaceutical Sciences, Key Laboratory of State Ministry of Education, Key Laboratory of Henan Province for Drug Quality Control and Evaluation, Collaborative Innovation Center of New Drug Research and Safety Evaluation, Zhengzhou University, 100 Kexue Avenue, Zhengzhou 450001, People's Republic of China

${ }^{4}$ Department of Biology and Biological Engineering, Chalmers University of Technology, SE-412 96 Gothenburg, Sweden

${ }^{5}$ Novo Nordisk Foundation Center for Biosustainability, Technical University of

Denmark, DK-2800 Kgs. Lyngby, Denmark

* Correspondence: xiaojunji@ njtech.edu.cn (X.-J. Ji)

\section{Highlights}

- Y. lipolytica is a superior industrial host for the production of lipids.

- Biosynthesis and degradation pathways of lipid in Y. lipolytica and the potential metabolic engineering targets are introduced.

- Metabolic engineering strategies for increasing lipid accumulation in Y. lipolytica are summarized.

- Multi-omics analysis, in silico metabolic models, and synthetic biology tools will further push the lipid yields to theoretical limits.

- Perspectives for novel engineering approaches for increasing lipid accumulation in $Y$. lipolytica are discussed. 


\begin{abstract}
Current energy security and climate change policies encourage the development and utilization of bioenergy. Oleaginous yeasts provide a particularly attractive platform for the sustainable production of biofuels and industrial chemicals due to their ability to accumulate high amounts of lipids. In particular, microbial lipids in the form of triacylglycerides (TAGs) produced from renewable feedstocks have attracted considerable attention because they can be directly used in the production of biodiesel and oleochemicals analogous to petrochemicals. As an oleaginous yeast that is generally regarded as safe, Yarrowia lipolytica has been extensively studied, with large amounts of data on its lipid metabolism, genetic tools, and genome sequencing and annotation. In this review, we highlight the newest strategies for increasing lipid accumulation using metabolic engineering and summarize the research advances on the overaccumulation of lipids in $Y$. lipolytica. Finally, perspectives for future engineering approaches are proposed.
\end{abstract}

\title{
Keywords
}

Yarrowia lipolytica, Metabolic engineering, Lipid accumulation, Triacylglycerides, Biodiesel 


\section{Table of Contents}

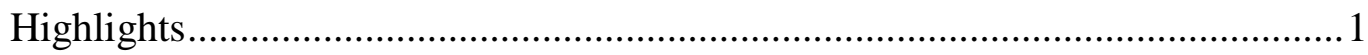

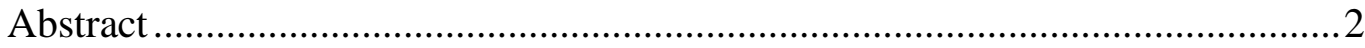

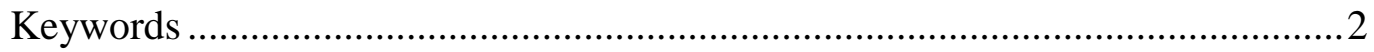

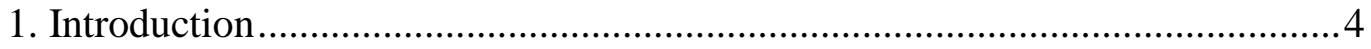

2. Biosynthesis and degradation of lipids in Y. lipolytica ....................................5

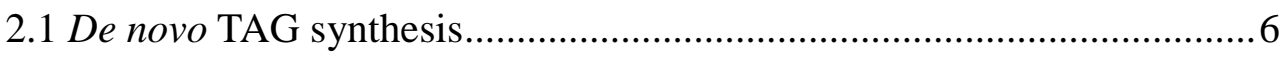

2.2 Ex novo TAG synthesis ................................................................. 7

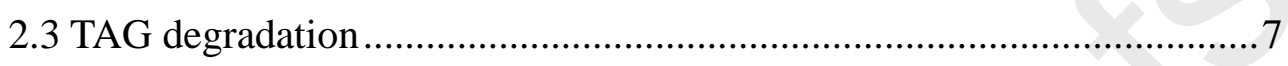

3. Enhancing lipid production through classical metabolic engineering .............. 7

3.1 Tuning endogenous genes involved in lipid metabolism ....................... 8

3.2 Tuning metabolic regulators involved in lipid metabolism .................... 8

3.3 Heterologous gene expression in Y. lipolytica ......................................

4. Novel strategies for enhancing lipid production in Y. lipolytica ......................10

4.1 Engineering alternative acetyl-CoA pathways .................................... 10

4.2 Engineering cytosolic NADPH pathways........................................... 11

4.3 Engineering oxidative stress defense pathways .................................. 11

4.4 Engineered fatty acid secretion pathways ........................................... 12

4.5 Adaptative evolution strategies ....................................................... 12

4.6 Computer-aided metabolic engineering ............................................ 13

5. Challenges and future perspectives ............................................................. 13

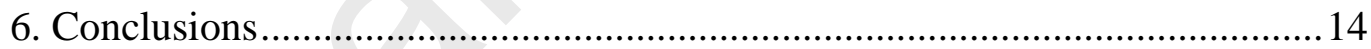

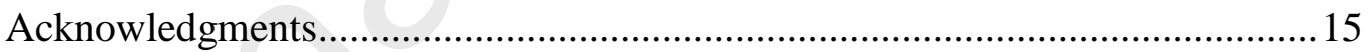

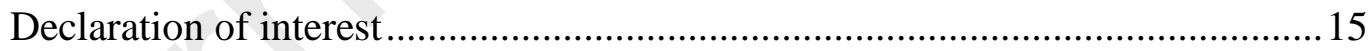

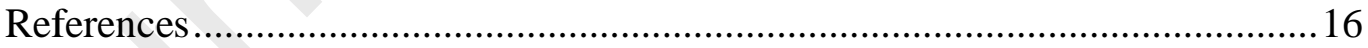




\section{Introduction}

Concerns about energy supply and the environment have driven the development and utilization of biofuels (Kim et al., 2019a). Recently, lipid-derived biodiesel has attracted widespread attention as a renewable fuel due to its high energy density, clean-burning properties, non-toxicity and biodegradablility (Ma et al., 2018). Yarrowia lipolytica is a non-conventional oleaginous yeast with "generally regarded as safe" (GRAS) status (Groenewald et al., 2014). Its genome has been sequenced and annotated (Liu et al., 2015a; Liu and Alper, 2014), metabolic pathways have been extensively studied (Dourou et al., 2018; Beopoulos et al., 2009a), and many genetic engineering tools have been developed (Larroude et al., 2018b; Shi et al., 2018; Gao et al., 2016). Moreover, Y. lipolytica can utilize a variety of inexpensive renewable substrates as carbon sources and can accommodate high flux of acetyl-CoA (Magdouli et al., 2017; Ledesma-Amaro and Nicaud, 2016a; Nambou et al., 2014; Poli et al., 2014). These characteristics make $Y$. lipolytica a remarkable industrial host for the production of many products, including lipid-derived biodiesel (Ledesma-Amaro and Nicaud, 2016b; Ledesma-Amaro, 2015); alkanes (Bruder et al., 2019); odd-chain fatty acids (Park et al., 2020; Park et al., 2018); plant-derived terpenoids, such as $\alpha$-farnesene (Liu et al., 2019c; Yang et al., 2016), carotenoids (Jacobsen et al., 2020; Larroude et al., 2018a), linalool (Cao et al., 2017), and others (Ma et al., 2019); organic acids, such as citric acid (Kamzolova and Morgunov, 2017), isocitric acid (Rzechonek et al., 2019), succinic acid (Li et al., 2018; Li et al., 2017), $\alpha$-ketoglutarate (Lei et al., 2019; Zeng et al., 2017), pyruvic acid (Kamzolova and Morgunov, 2018) and crotonic acid (Wang et al., 2019); as well as sugar alcohols, such as erythritol (Liu et al., 2019b; Liu et al., 2017c), erythrulose (Carly et al., 2018), isomaltulose (Zhang et al., 2018). Even more value-added functional polyunsaturated fatty acids (PUFAs), such as $\gamma$-linolenic acid (Sun et al., 2017), arachidonic acid (Liu et al., 2019a; Liu et al., 2017a; Liu et al., 2017b), eicosapentaenoic acid (Xue et al., 2013), and docosahexaenoic acid (Gemperlein et al., 2019) were produced in engineered $Y$. lipolytica strains.

Metabolic engineering strategies for increasing lipid accumulation in $Y$. lipolytica have expanded considerably. The early efforts focused on: (i) Maximizing the flux towards lipid biosynthesis, such as deleting genes related to $\beta$-oxidation involved in the lipid degradation pathway (Dulermo and Nicaud, 2011), tuning the activity of transcription factors involved in lipid metabolism (Leplat et al., 2018), overexpressing fatty acid (FA) and triacylglyceride (TAG) synthesis genes (Tai and Stephanopoulos, 2013), overexpressing functional heterologous lipid synthetic related genes in $Y$. lipolytica (Friedlander et al., 2016; Zhang et al., 2014). (ii) Minimizing the flux towards competing metabolic pathways, such as the inhibition of glycogen and citric acid biosynthesis pathways (Bhutada et al., 2017; Sagnak et al., 2018). The current novel strategies focus on: (i) Expanding the range of available substrates (Johnravindar et al., 2018; Ledesma-Amaro and Nicaud, 2016a; Dobrowolski et al., 2016; Sestric et al., 2014);

(ii) Designing new approaches to secrete intracellular fatty acids into the culture medium (Ledesma-Amaro et al., 2016a); (iii) Engineering alternative acetyl-CoA pathways to decouple nitrogen limitation, enabling $Y$. lipolytica to produce large amounts of acetyl- 
CoA and accumulate lipids even in the presence of nitrogen (Xu et al., 2016); (iv) Engineering cytosolic NADPH pathways to provide more NADPH (Qiao et al., 2017); (v) Engineering oxidative stress defense pathways to mitigate lipotoxicity and improve cell morphology (Xu et al., 2017b); (vi) Evolutionary metabolic engineering combining adaptive evolution with metabolic engineering, resulting in strains that have both high biomass yields and high lipid production capacity (Liu et al., 2015c); (vii) Screening different metabolic modification targets based on predictions made using genome-scale metabolic models (GEMs) (Kim et al., 2019b). In order to assess the extent to which different engineering approaches increase lipid production, the theoretical limits of process yield must first be determined. Papanikolaou et al. (2011) used stoichiometric ratios to calculate the theoretical limits of lipid production from different substrates. They indicated that in terms of glucose (and similar sugars such as lactose, fructose, etc), about 1.1 moles of acetyl-CoA are generated from 100 grams of glucose ( 0.56 moles) catabolized. If all the acetyl-CoA produced is channeled towards lipid synthesis, the maximum theoretical yield of lipid produced per gram of glucose consumed is around $0.32 \mathrm{~g} / \mathrm{g}$. Similarly, xylose can be either catabolized by the phosphoketolase reaction, which is the most efficient pathway producing around 1.2 moles of acetyl-CoA per 100 grams of xylose ( $\sim 0.66$ moles) utilized, or through the pentose phosphate pathway, where around 1.0 mole of acetyl-CoA is formed per 100 grams of xylose utilized. Assuming that oleaginous microorganisms utilize exclusively the phosphoketolase pathway to assimilate xylose, the maximum theoretical yield of lipid produced per gram of xylose consumed is around $0.34 \mathrm{~g} / \mathrm{g}$. As far as glycerol is concerned, the maximum theoretical yield of lipid is around $0.30 \mathrm{~g} / \mathrm{g}$.

In this review, we summarize the recent research advances on lipid accumulation in $Y$. lipolytica. We first introduced the lipid biosynthesis and degradation pathways of $Y$. lipolytica and the potential metabolic engineering targets. Then, we focus on strategies for increasing lipid accumulation in Y. lipolytica using metabolic engineering. Finally, we propose future perspectives for new engineering approaches.

\section{Biosynthesis and degradation of lipids in Y. lipolytica}

Intracellular lipids include neutral lipids (TAGs and steryl esters) and non-neutral lipids (free fatty acids (FFAs), glycolipids, and phospholipids). In general, the former serve as an energy reserve for the cell, while the latter perform special physiological functions (Darvishi et al., 2017). Approximately 95\% of the intracellular lipids in $Y$. lipolytica are stored in lipid bodies (LBs) in the form of TAGs (Zeng et al., 2018). TAGs can be synthesized via two pathways (Papanikolaou and Aggelis, 2011): (i) de novo synthesis, which requires hydrophilic substrates such as sugars, organic acids, or alcohols to produce FA precursors, primarily acetyl-CoA, and integrates them into the Kennedy pathway to synthesize TAGs; (ii) ex novo biosynthesis, which involves the hydrolysis of hydrophobic substrates (such as alkanes, FFAs, and TAGs) to produce FAs and glycerol, and transports them inside the cell to reassemble them into TAGs. 


\subsection{De novo TAG synthesis}

The de novo TAG synthesis pathway is stimulated when nitrogen is depleted and carbon is abundant in the medium (Mathiazhakan et al., 2016). The nitrogen depletion process involves a series of regulatory events related to the central metabolism. Initially, the nitrogen depletion leads to increased activity of adenosine monophosphate (AMP) deaminase (AMPD), which converts AMP into inosine monophosphate (IMP) and ammonia (Evans et al., 1983). Isocitrate dehydrogenase $(I D H)$ is an AMP-dependent dehydrogenase that is inhibited by low AMP concentrations (Botham and Ratledge, 1979). As a result, the tricarboxylic acid (TCA) cycle is downregulated, and the carbon flux from isocitric acid no longer flows toward $\alpha$-ketoglutarate. The citric acid accumulated in the mitochondria is transported into the cytosol by malate/citrate transferase in the mitochondrial membrane (Palmieri et al., 1996). Subsequently, citrate is cleaved by ATPcitrate lyase (ACL1 and ACL2) to produce acetyl-CoA and oxaloacetate. In this way, $Y$. lipolytica can produce abundant cytosolic acetyl-CoA, which is the direct precursor of FA biosynthesis (Figure 1).

FA biosynthesis in the cytosol involves a series of reactions that convert the precursor acetyl-CoA to long-chain fatty acids. Firstly, malonyl-CoA is generated by carboxylation of acetyl-CoA with acetyl-CoA carboxylase (ACC1) (Al-Feel et al., 1992). Then, acetylCoA and malonyl-CoA are condensed to acyl-CoA under the action of the FA synthase complex (FAS1 and FAS2). The FA synthase use NADPH as the reducing cofactor, and each step in the elongation of the acyl-CoA chain requires two NADPH molecules. The chain length of naturally synthesized acyl-CoAs is typically 16 or 18 carbon atoms. In the next step, these C16:0 and C18:0 molecules are delivered to the endoplasmic reticulum (ER) for further elongation and desaturation (Ledesma-Amaro and Nicaud, 2016b) (Figure 1).

TAG synthesis generally follows the Kennedy pathway, which uses acyl-CoA and glycerol-3-phosphate (G3P) as the direct substrates (Papanikolaou and Aggelis, 2003). TAG assembly begins with the action of G3P acyltransferase (SCT1), which catalyzes the conversion of G3P to lysophosphatidic acid (LPA) (Beopoulos et al., 2008). LPA is then further acylated by LPA acyltransferase ( $S L C 1$ ) to generate phosphatidic acid (PA). PA is further dephosphorylated to diacylglycerol (DAG) by phosphatidic acid phosphatase $(P A P)$ (Silverman et al., 2016). In the final step of TAG assembly, DAG is acylated at the sn-3 position, either by an acyl-CoA-dependent or acyl-CoA-independent reaction, to form TAG (Tai and Stephanopoulos, 2013; Beopoulos et al., 2009b). The acyl-CoAdependent reaction is catalyzed by DAG acyltransferases (DGA1 or DGA2), with acyl$\mathrm{CoA}$ as the final acyl group donor. The acyl-CoA-independent reaction is catalyzed by phospholipid DAG acyltransferase (LRO1), which uses glycerophospholipid as the acyl group donor (Figure 1). Moreover, steryl ester synthetase encoded by ARE1 has been demonstrated to promote DAG acylation by acting as acyltransferase in an acyl-CoAdependent reaction. The primary enzymes involved in these pathways are summarized in Table 1. 


\subsection{Ex novo TAG synthesis}

Y. lipolytica can also utilize hydrophobic substrates such as TAGs, alkanes, and FFAs to synthesize lipids, and this pathway requires the hydrolysis of hydrophobic substrates. TAGs are hydrolyzed by a large battery of extracellular lipases, which release FAs and glycerol for cellular uptake (Dulermo et al., 2015b). Alkanes entering the cell are hydroxylated in the endoplasmic reticulum (ER) using the cytochrome P450-dependent alkane monooxygenases. The thus formed corresponding fatty alcohols are oxidized to aldehydes by dehydrogenases and further to FAs by long-chain-aldehyde oxidases (Fukuda, 2013). The released FAs can be activated by binding to coenzyme A into acylCoAs through the acyl-CoA synthetase (FAA1), which are then reassembled into TAGs in the ER. Both the de novo and ex novo TAG synthesis pathways are summarized in Figure 1.

\subsection{TAG degradation}

The accumulated intracellular TAGs stored in LBs serve as an energy reserve for the cell. Once carbon is insufficient, the TAG will be degraded to release acetyl-CoA for maintaining cellular metabolism. Initially, FFAs can be released from TAGs under the action of the intracellular lipases TGL3 and TGL4 (Dulermo et al., 2013). The released FFAs can be activated to acyl-CoAs by the enzyme FAAl and then transported into the peroxisome by the specific transporters Pxa1 and Pxa2 (Dulermo et al., 2015b). Alternatively, the released FFAs can also be transported into the peroxisome and then activated there to acyl-CoAs by acyl/aryl-CoA ligase ( $A A L$ ) (Dulermo et al., 2016). Subsequently, acyl-CoAs are degraded through the $\beta$-oxidation pathway in the peroxisomes to release acetyl-CoA. Cyclical $\beta$-oxidation encompasses the four steps of oxidation, hydration, dehydrogenation, and thiolysis, which are repeated for each $\mathrm{C} 2$ unit. Six acyl-CoA oxidases (POX1-6) catalyze the first step, while the multifunctional enzyme MFE1 catalyzes the second and third steps (Mlickova et al., 2004). The last step is catalyzed by 3-ketoacyl-CoA thiolase (POT1) (Berninger et al., 1993). Because $\beta$ oxidation occurs in peroxisomes, peroxisome biogenesis is usually downregulated by deleting PEX3, PEX10, and PEX11 to prevent the degradation of TAGs in industrial strains (Dulermo et al., 2015a; Xue et al., 2013). Additionally, genes related to the $\beta$ oxidation pathway are also often targets for deletion to increase lipid accumulation (Lazar et al., 2018).

\section{Enhancing lipid production through classical metabolic engineering}

The ability of $Y$. lipolytica to accumulate lipids can be improved by increasing the biosynthetic precursors (such as acetyl-CoA, malonyl-CoA, NADPH, ATP), shuting down the degradation pathways, and adjusting the fermentation conditions (such as culture medium, $\mathrm{C} / \mathrm{N}$ ratio, $\mathrm{pH}$, inoculation volume, fermentation mode). With the development of metabolic engineering and synthetic biology, the biosynthesis of lipids has been further improved by optimizing the complex metabolic network of Y. lipolytica. In the past few years, researchers used a variety of approaches to increase lipid production 
in $Y$. lipolytica. These metabolic engineering strategies are summarized in Table 2.

\subsection{Tuning endogenous genes involved in lipid metabolism}

Lipid-overaccumulation phenotypes can be produced by combining the overexpression of genes related to lipid biosynthesis with the deletion of genes related to lipid degradation (Dulermo and Nicaud, 2011). For instance, Lazar et al. (2014) combined the deletion of POX1-6 and TGL4 with the overexpression of GDP1 and DGA2, and the resulting strain JMY3501 showed reduced FA degradation and increased intracellular G3P concentration, which improved TAG accumulation. Blazeck et al. (2014) used a combinatorial strategy to synergistically regulate multiple key genes involved in lipid degradation and biosynthesis in Y. lipolytica, including PEX10, MFE1, AMPD, ACL1, $A C L 2, M A E, D G A 1$, and $D G A 2$, which generated 57 distinct genotypes. The most effective modifications were the double deletion of PEX10 and MFE1 and the overexpression of $D G A 1$. After optimizing the bioreactor conditions, the engineered strain produced a lipid titer of 25 grams of lipid per liter of culture, with a lipid content of $71 \%$ in cell dry weight, representing a 60 -fold improvement over the starting strain. Tai and Stephanopoulos (2013) adopted a push-and-pull strategy by overexpressing $A C C 1$ and DGA1 simultaneously. Compared with the starting strain Po1g, the engineered strain exhibited an increase of the lipid content from 8.7 to $41 \%$. After $120 \mathrm{~h}$ of fermentation in a 2-L bioreactor, the lipid content of the engineered strain reached $61.7 \%$, with a lipid yield of 0.195 grams of lipid per gram of carbon substrate consumed, and lipid productivity of $0.143 \mathrm{~g} /(\mathrm{L} \cdot \mathrm{h}$ ) (grams of lipid per liter of culture per hour). Qiao et al. (2015) adopted a promoter engineering strategy by replacing hp4d with the TEFin promoter to co-overexpress $A C C 1$ and $D G A 1$ and overexpress the stearoyl-CoA desaturase $(S C D)$ in $Y$. lipolytica. In a 1.5-L bioreactor with glucose as the substrate, the engineered strain produced a lipid content and titer of $67 \%$ and $55 \mathrm{~g} / \mathrm{L}$, respectively. It is possible that the overexpression of $S C D$ can release the potential allosteric inhibition of $A C C l$ by saturated fatty acids and increase fatty acid biosynthetic flux by continuously converting inhibitory saturated fatty acids into monounsaturated fatty acids (Flowers and Ntambi, 2008). Moreover, the engineered strain also exhibited a variety of desirable phenotypes, such as fast growth, high sugar tolerance, and high unsaturated fatty acid content (Qiao et al., 2015). Finally, channeling the metabolic flux towards lipid synthesis can also improve lipid accumulation to a certain extent. Bhutada et al. (2017) identified and deleted the GSY1 gene encoding a glycogen synthetase, and TAG accumulation increased by $60 \%$ compared with the wild-type strains, proving that glycogen synthesis is a competing pathway whose elimination promotes the production of neutral lipids.

\subsection{Tuning metabolic regulators involved in lipid metabolism}

The tuning of cellular metabolic regulators can effectively enhance the carbon flux and facilitate the accumulation of lipids (Leplat et al., 2018; Wang et al., 2018). For instance, the glucose repression regulator $S N F 1$ is involved in the transition from the growth phase to the oleaginous phase, and can allow the strain to accumulate high levels of lipids even in the presence of nitrogen. Seip et al. (2013) deleted the SNF1, resulting 
in 2.6-fold higher lipid content than in the wild-type strain. Similarly, the deletion of the glucose repression regulator $M I G 1$ enhanced the lipid content from 36 to $48.7 \%$. The authors observed that the deletion of MIG1 resulted in the downregulation of MFE1 transcription and the upregulation of many genes related to lipid biosynthesis. These changes of the gene expression profile might be responsible for the increased LB formation and lipid content in the gene deletion strain (Wang et al., 2013). In a separate study, Liu et al. (2015b) sequenced the previous evolved strains E13 and E26 and identified the lipogenesis factor YALIOF26191g (UGA2), which encodes succinate semialdehyde dehydrogenase that takes part in the gamma-aminobutyric acid (GABA) assimilation pathway. The deletion of $U G A 2$ enhanced the lipid content by $50 \%$ compared to the starting strain (Polf $\Delta$ pex $10 \Delta m f e$ ). The reduction in GABA assimilation could lead to an imbalance in the intermediates of the TCA cycle, thereby reducing the flux through the TCA cycle and channeling more carbon towards the lipogenic pathway. In another similar study, Liu et al. (2015c) discovered that Mga2p is a regulator of desaturase gene expression and an effective lipogenesis factor that is involved in regulating the metabolism of unsaturated fatty acids in $Y$. lipolytica. The authors introduced a single amino acid mutation into $M g a 2 p$ and combined it with previous metabolic modifications to obtain a producer strain with a lipid titer of $25 \mathrm{~g} / \mathrm{L}$, which showed an imbalance between glycolysis and the TCA cycle can be used as a driving force for lipogenesis.

\subsection{Heterologous gene expression in Y. lipolytica}

Heterologous gene expression in Y. lipolytica was successfully used to increase lipid accumulation. For instance, Friedlander et al. (2016) combined the overexpression of DGA1 from Rhodosporidium toruloides and DGA2 from Claviceps purpurea with the deletion of the endogenous TGL3, and the resulting strain showed a lipid content of $77 \%$ and a lipid yield of $0.21 \mathrm{~g} / \mathrm{g}$ in batch fermentation. Similar modifications, combining the overexpression of heterologous $\Delta-15$ desaturase $(\triangle-15 D)$ from flax and endogenous genes (ACC1,DGA1, SCD , $\triangle-12 D$ ) with the deletion of the endogenous PEX10 and MFE1, led to a superior lipid producer with a lipid content and titer of $77.8 \%$ and $50 \mathrm{~g} / \mathrm{L}$, respectively, using glucose as substrate in a 5-L stirred-tank bioreactor (Yan et al., 2020). Although $A C L$ is considered to be the key enzyme for generating acetyl-CoA in oleaginous yeasts, the overexpression of the native $A C L$ gene did not significantly increase the lipid content of $Y$. lipolytica (Blazeck et al., 2014). Notably, the native $A C L$ has a high $K_{M}$ value of 3.6 $\mathrm{mM}$, which means that it has a low affinity for the substrate. Consequently, a heterologous ACL from Mus musculus, with a much lower $K_{\mathrm{M}}$ value for citrate of $0.05 \mathrm{mM}$, was overexpressed in Y. lipolytica, which improved the lipid content from 7.3 to $23.1 \%$ (Zhang et al., 2014). Similarly, the heterologous expression of hemoglobin from Vitreoscilla in $Y$. lipolytica increased the utilization of oxygen by the strain during fermentation, thereby improving the growth and increasing the final lipid yield. This modification resulted in an increase in the lipid content by $40 \%$ compared with the parental strain when dissolved oxygen (DO) was controlled at $30 \%$ of the atmospheric value (Zhang et al., 2019). In a separate study, Walker et al. (2020) investigated the effect of thiamine on the physiology of $Y$. lipolytica and found that thiamine deficiency decreased the protein abundance related to the lipid biosynthesis pathways and energy metabolism. The authors further 
used the thiamine-regulated promoter P3 to express 4-amino-5-hydroxymethyl-2methylpyrimidine phosphate synthase (THI13) from $S$. cerevisiae and proved that restoring thiamine synthesis can boost lipid accumulation in $Y$. lipolytica.

\section{Novel strategies for enhancing lipid production in Y. lipolytica}

In addition to the regulation of the target gene listed above, several novel strategies have emerged in the past few years. These studies emphasize a deeper understanding of the limiting factors and bottlenecks in lipogenesis and rely on the design of reasonable approaches to eliminate these effects. Different approaches for increasing lipid accumulation in Y. lipolytica are summarized in Figure 2.

\subsection{Engineering alternative acetyl-CoA pathways}

In Y. lipolytica, lipogenic acetyl-CoA is only generated by the splitting of citrate by ATP-citrate lyase when the TCA cycle is repressed under nitrogen-limited conditions (Beopoulos et al., 2009b). Therefore, the availability of acetyl-CoA limits lipid biosynthesis. To decouple acetyl-CoA formation from nitrogen starvation and enhance acetyl-CoA supply, Xu et al. (2016) proposed five effective approaches based on establishing an alternative cytosolic acetyl-CoA formation pathway to decouple lipid synthesis from nitrogen starvation. One of the most efficient approaches was the overexpression of heterologous peroxisomal carnitine acetyltransferase (perCAT2) from $S$. cerevisiae and endogenous genes (ACC1, DGA1). The results showed that perCAT2 improved both the lipid titer and content compared with the starting strain with $A C C 1$ and

DGA1 overexpression. They also overexpressed the phosphoketolasephosphotransacetylase (PK-Pta) pathway to couple NADPH regeneration with cytosolic acetyl-CoA formation, which increased the lipid yield to $0.225 \mathrm{~g} / \mathrm{g}$ in shake-flask cultures. When cultured in a 3-L bioreactor, the perCAT2 overexpression strain produced $66.4 \mathrm{~g} / \mathrm{L}$ of lipids with a yield of $0.229 \mathrm{~g} / \mathrm{g}$ of glucose and productivity of $0.565 \mathrm{~g} /(\mathrm{L} \cdot \mathrm{h})$, representing a 3.1-fold increase of lipid production compared with the starting strain. Additionally, the engineered strains accumulated lipids at the initial stages of growth and continued throughout the entire fermentation process. In another study, Niehus et al. (2018) overexpressed the endogenous xylitol dehydrogenase $(X D H)$, xylose reductase $(X R)$, and xylulose kinase $(X K)$ in the Pold strain, which allowed it to grow with xylose as the sole carbon source. The authors further designed two additional pathways to generate acetyl-CoA from xylulose-5-P, and both pathways increased lipid production from xylose. One of the most efficient approaches based on overexpressing heterologous phosphoketolase $(X P K A)$ and acetate kinase $(A C K)$, which results in the conversion of xylose to acetate, which is further converted to lipogenic acetyl-CoA by acetyl-CoA synthetase $(A C S)$. The resulting strain produced a lipid content of $67 \%$, lipid titer of 16.5 $\mathrm{g} / \mathrm{L}$, lipid yield of $0.344 \mathrm{~g} / \mathrm{g}$, and lipid productivity of $1.85 \mathrm{~g} /(\mathrm{L} \cdot \mathrm{h})$ in a fed-batch bioreactor using lignocellulosic hydrolysate as the sole carbon source. 


\subsection{Engineering cytosolic NADPH pathways}

To better understand the mechanisms and bottlenecks of lipid metabolism, the gene encoding malic enzyme of $Y$. lipolytica was expressed in Escherichia coli, indicating that malic enzyme used $\mathrm{NAD}^{+}$as the major cofactor. Furthermore, when the $\mathrm{NADP}^{+}$ dependent malic enzyme from Mortierella alpina (MCE2) was expressed in Y. lipolytica, the resulting strain showed no significant changes in lipid content or fatty acid composition, which indicated that the malic enzyme is not the primary source of NADPH for lipogenesis in Y. lipolytica (Zhang et al., 2013). Wasylenko et al. (2015) conducted ${ }^{13} \mathrm{C}$ metabolic flux analysis (MFA) of $Y$. lipolytica grown with glucose as the carbon source. By comparing the flux distribution of wild-type and engineered strains, the authors concluded that the oxidative pentose phosphate pathway (oxPPP) is the major NADPH source for lipogenesis. Qiao et al. (2017) established a quantitative model to analyze the global metabolic network during lipid accumulation. After verification and sensitivity analysis, it was found that the supply of NADPH limited lipogenesis, suggesting lipid accumulation can be improved by increasing the supply of NADPH (Ji and Huang, 2019). Qiao et al. (2017) then designed four engineered biosynthetic pathways to convert glycolytic NADH into cytosolic NADPH. The first strategy was overexpression of two NADP ${ }^{+}$-dependent G3P dehydrogenases, GapC from Clostridium acetobutylicum and GPD1 from Kluyveromyces lactis, which respectively increased the lipid yield by 20.0 and $17.8 \%$. The second strategy was activating the pyruvate/oxaloacetate/malate (POM) cycle by cloning and expressing MCE2 from Mucor circinelloides in $\mathrm{AD}$ strains (overexpression of $A C C 1$ and DGAl), which increased the lipid yield from 0.17 to $0.21 \mathrm{~g} / \mathrm{g}$. The third approach was co-expression of a phosphoketolase from Leuconostoc mesenteroides and a phosphate acetyltransferase from Clostridium kluyveri in AD strain to activate the NOG pathway, which can generate 3 mol acetyl-CoA from $1 \mathrm{~mol}$ glucose to increase the supply of acetyl-CoA. The engineered strain showed a $16 \%$ increase in lipid content. Finally, co-expression of a heterologous GapC and a heterologous MCE2 further increased the lipid yield to 0.231 $\mathrm{g} / \mathrm{g}$. In an optimized bioreactor, the highest lipid titer, productivity, and yield reached 99.3 $\mathrm{g} / \mathrm{L}, 1.2 \mathrm{~g} /(\mathrm{L} \cdot \mathrm{h})$, and $0.279 \mathrm{~g} / \mathrm{g}$, respectively.

\subsection{Engineering oxidative stress defense pathways}

Lipid accumulation in oleaginous yeasts is stimulated by nitrogen starvation, which triggers global physiological rearrangements and a variety of cellular stress responses (Rosenwasser et al., 2014; Morin et al., 2011). One of the main stress factors during lipid accumulation is the increased concentration of reactive oxygen species (ROS) due to lipid oxidation and peroxidation (Zhang et al., 2020; Li et al., 2011). ROS can attack the nucleophilic centers of many bioactive molecules (such as DNA, proteins, and lipids) and cause oxidative damage (Shi et al., 2017), triggering a series of cellular stress events, including cellular aging (Zimniak, 2011), replicative DNA damage (Mano et al., 2002), and protein adducts (Grimsrud et al., 2008). As natural molecules with fatty acids as the main components, neutral lipids are vulnerable to be attacked by oxygen free radicals through a chain reaction mechanism, and the resulting lipid peroxides can undergo further 
autolysis to produce reactive aldehydes (Xu et al., 2017b). The carbonylation caused by reactive aldehydes attacks lysine, histidine, and cysteine residues, which inactivates critical enzymes of lipid metabolism, resulting in reduced cell viability and inefficiency of biosynthetic pathways (Grimsrud et al., 2008). Moreover, increased oxidative stress may result in the consumption of stored lipids as an energy source to maintain cellular homeostasis (Shi et al., 2017). Therefore, Xu et al. (2017b) adopted a metabolic engineering strategy by overexpressing glutathione peroxidase $(y l G P O)$, glutathione disulfide reductase $(y l G S R)$, and glucose-6-phosphate dehydrogenase (ScZwf) to upregulate the oxidative stress defense pathways, while simultaneously overexpressing aldehyde dehydrogenase $(E c A l d H)$ to detoxify reactive aldehydes, which could effectively increase the cellular resistance to oxidative stress and improve cell morphology. The resulting strain showed a lipid content of $81.4 \%$, a lipid titer of $72.7 \mathrm{~g} / \mathrm{L}$, and lipid productivity of $0.97 \mathrm{~g} /(\mathrm{L} \cdot \mathrm{h})$ in a controlled bench-top bioreactor. These results indicate that engineering the oxidative defense pathway is vital for increasing lipid accumulation.

\subsection{Engineered fatty acid secretion pathways}

The high cost of recovery and separation processes often limits the economics of microbial lipid production (Ji and Ledesma-Amaro, 2020; Meullemiestre et al., 2016). To overcome these limitations, Ledesma-Amaro et al. (2016a) focused on designing unique approaches to secrete FAs into the culture medium, followed by an in situ separation method using organic layers. They engineered two synthetic pathways to achieve these aims. The first strategy enhanced FFA flux by combining the overexpression of genes involved in TAG synthesis and degradation (DGA2, TGLA, and KlTGL3) with the deletion of genes involved in FFA activation and degradation (FAAl and MFE1), which resulting in FFA accumulation and subsequent secretion. The second strategy aimed to abolish LB formation altogether and redirect FA synthesis to the cytosol by mimicking bacterial pathways with re-localized acyl-CoA thioesterases. They engineered a strain by deleting four genes (ARE1, DGA1, DGA2, and LRO1) to completely abolish the formation of neutral lipids (TAG and SE), combined with the deletion of FAAl and MFE1, as well as the overexpression of RnTEII, which increased the secretion of FAs. After using an optimized bioreactor to cultivate the best engineered strains, a titer of secreted FAs 10.4 $\mathrm{g} / \mathrm{L}$, a yield of $0.20 \mathrm{~g} / \mathrm{g}$, and total equivalent lipid content of $120.4 \%$ of the DCW was achieved, which significantly exceeded the storage limit of individual cells. These synthetic approaches decoupled production from biomass formation and facilitated product extraction, which may be a significant breakthrough for overcoming current limitations.

\subsection{Adaptative evolution strategies}

Rational metabolic engineering often results in a decrease of biomass while increasing product yield, and therefore compromises overall productivity (Trentacoste et al., 2013). Evolutionary metabolic engineering may provide a balance between these two competing factors (Abatemarco et al., 2013). For instance, Liu et al. (2015b) established 
a rapid evolutionary metabolic engineering method based on floating cell enrichment screening. The authors observed that when the culture was stabilized, cells with high lipid content could float on top of the culture medium, and normal cells settled to the bottom. Here, the authors used ethyl methanesulfonate (EMS) to mutate floating cells randomly, screened mutants with higher lipid content by mild centrifugation (e.g., $100 \times \mathrm{g}$ ), and finally measured the lipid content and cell growth ability. This iterative process increased the lipid titer, productivity, and yield to $39.1 \mathrm{~g} / \mathrm{L}, 0.56 \mathrm{~g} /(\mathrm{L} \cdot \mathrm{h})$, and $0.244 \mathrm{~g} / \mathrm{g}$, respectively. Importantly, the floating cell enrichment scheme resulted in increased lipid production without reducing biomass. Moreover, the evolved strain exhibited lipid accumulation during the cell growth phase without the need for nitrogen starvation. In another study, Daskalaki et al. (2019) adopted an adaptive laboratory evolution strategy to improve lipogenesis by alternately changing the culture medium and continuously screening highlipid cells, which ultimately coupled growth with lipid accumulation. Strains developed after 77 generations had a lipid content of $44 \%$, which was $30 \%$ higher than that of the starting strain. Therefore, evolutionary strategies can boost lipid accumulation without affecting biomass, and combined with metabolic engineering and multi-omics technologies can maximize the robustness and productivity of oleaginous yeast.

\subsection{Computer-aided metabolic engineering}

By using in silico modeling to guide metabolic engineering, new strains with high lipid accumulation have been engineered (Xu et al., 2020; Kerkhoven et al., 2016; Kavšček et al., 2015; Pan and Hua, 2012). For instance, Kim et al. (2019b) used a genome-scale model of metabolism (GEM) to identify potential genetic engineering targets for increasing lipid production in $Y$. lipolytica. Several overexpression and knockout targets that might increase lipid accumulation were identified, including diglyceride acyltransferase, acetyl-CoA carboxylase, and stearoyl-CoA desaturase et al. One of the knockout targets YALIOF30745g, encoding an enzyme involved in onecarbon/methionine metabolism, was experimentally validated. The resulting mutant showed increased the lipid content by $45 \%$ compared to the wild-type. Similarly, Wei et al. (2017) applied another GEM to predict six gene amplification targets that could enhance the precursor supply of acetyl-CoA and malonyl-CoA, and lead to $34.1 \%$ increasement of TAG biosynthesis. Moreover, the simulations showed that supplementation of six amino acids in growth medium, including L-glycine, L-alanine, L-cysteine, L-serine, L-threonine, and L-aspartic acid, could increase TAG accumulation. Among them, the addition of L-threonine and L-aspartic acid might increase the yield of TAG by $55.5 \%$. In conclusion, such mathematical modeling approach may provide an attractive platform for better understanding of $Y$. lipolytica metabolic network and guiding the rational metabolic engineering.

\section{Challenges and future perspectives}

Although substantial improvements of lipid production have been achieved with $Y$. lipolytica, high costs still limited the economic benefits of biofuels and oleochemicals derived from microbial lipids. Consequently, inexpensive substrates, such as 
lignocellulose and industrial by-products, were often used to minimize production costs in industrial-scale plant. However, toxic compounds present in inexpensive substrates would be potential inhibitors that can lead to unacceptably low growth rates and limit the industrialization of current processes. To this end, it is possible to improve the tolerance of engineered strains to toxic compounds by designing detoxification pathways or adding antidotes. Therefore, the use of inexpensive industrial composite substrates should be a considerable direction in future applied research. Furthermore, the third-generation biofuels aim to utilize microbial cell factories to convert $\mathrm{CO}_{2}$ into fuels and chemicals. Future research directions should consider using $Y$. lipolytica to produce lipid directly from $\mathrm{CO}_{2}$, including artificial photosynthesis, carbon fixation and utilization, feedstock tolerance, and strain process engineering.

Recent developments in systems and synthetic biology have made it possible to transform living organisms to serve humanity. Constructing an efficient cell factory based on $Y$. lipolytica requires the combination of multi-omics technologies to understand the regulatory mechanisms and bottlenecks in lipid metabolism, the guidance of in silico metabolic models to design and optimize more efficient synthetic pathways, and the development of synthetic biology tools to assemble and fine-tune $Y$. lipolytica metabolism, so that the precursors in the metabolic pathway are adequately supplied, the metabolic fluxes and cofactors are balanced, and feedback inhibition is alleviated, which would further push the lipid production towards the theoretical maximum limits. We believe that with the advancement of synthetic biology, more novel tools and regulatory methods will be developed, which would accelerate the construction of $Y$. lipolytica cell factories with desired lipogenesis phenotype.

\section{Conclusions}

Synthesis of microbial lipids from renewable feedstocks is one of the most promising strategies for biodiesel and oleochemicals production. Metabolic engineering of $Y$. lipolytica for increasing lipid accumulation has achieved considerable success. Future approaches to further increase lipid production using glucose or other inexpensive substrates in $Y$. lipolytica are expected to require the combination of systems metabolic engineering, multi-omics integration analysis, computer-aided designs, and synthetic biology approaches, which would further push the lipid production towards theoretical maximum limits. 


\section{Acknowledgments}

This work was financially supported by the National Science Fund for Excellent Young Scholars of China (No. 21922806), the National Key Research and Development Program of China (No. 2018YFA0903700), the National Natural Science Foundation of China (Nos. 21776131, 31800079), the Six Talent Peaks Project in Jiangsu Province of China (No. 2018-SWYY-047), the Jiangsu Synergetic Innovation Center for Advanced BioManufacture (No. XTD1814), and the BBSRC grant BB/R01602X/1.

\section{Declaration of interest}

We declare that we have no financial and personal relationships with other people or organizations that can inappropriately influence our work, there is no professional or other personal interest of any nature or kind in any product, service and/or company that could be construed as influencing the position presented in, or the review of, the manuscript entitled, "Metabolic engineering for increased lipid accumulation in Yarrowia lipolytica". 


\section{References}

1. Abatemarco, J., Hill, A., Alper, H.S. 2013. Expanding the metabolic engineering toolbox with directed evolution. Biotechnol. J. 8(12), 1397-1410.

2. Al-Feel, W., Chirala, S.S., Wakil, S.J. 1992. Cloning of the yeast FAS3 gene and primary structure of yeast acetyl-CoA carboxylase. Proc. Natl. Acad. Sci. U. S. A. 89(10), 4534.

3. Beopoulos, A., Cescut, J., Haddouche, R., Uribelarrea, J.L., Molina-Jouve, C., Nicaud, J.M. 2009a. Yarrowia lipolytica as a model for bio-oil production. Prog. Lipid Res. 48(6), 375-87.

4. Beopoulos, A., Chardot, T., Nicaud, J.-M. 2009b. Yarrowia lipolytica: A model and a tool to understand the mechanisms implicated in lipid accumulation. Biochimie 91(6), 692-696.

5. Beopoulos, A., Mrozova, Z., Thevenieau, F., Le Dall, M.T., Hapala, I., Papanikolaou, S., et al. 2008. Control of Lipid Accumulation in the Yeast Yarrowia lipolytica. Appl. Environ. Microbiol. 74(24), 7779-7789.

6. Berninger, G., Schmidtchen, R., Casel, G., Knorr, A., Rautenstrauss, K., Kunau, W.H., et al. 1993. Structure and metabolic control of the Yarrowia lipolytica peroxisomal 3-oxoacyl-CoA-thiolase gene. Eur. J. Biochem. 216(2), 607-613.

7. Bhutada, G., Kavscek, M., Ledesma-Amaro, R., Thomas, S., Rechberger, G.N., Nicaud, J.M., et al. 2017. Sugar versus fat: elimination of glycogen storage improves lipid accumulation in Yarrowia lipolytica. FEMS Yeast Res. 17(3).

8. Blazeck, J., Hill, A., Liu, L., Knight, R., Miller, J., Pan, A., et al. 2014. Harnessing Yarrowia lipolytica lipogenesis to create a platform for lipid and biofuel production. Nat. Commun. 5, 3131.

9. Botham, P.A., Ratledge, C. 1979. A Biochemical Explanation for Lipid Accumulation in Candida 107 and Other Oleaginous Micro-organisms. Microbiology 114(2), 361-375.

10. Bruder, S., Moldenhauer, E.J., Lemke, R.D., Ledesma-Amaro, R., Kabisch, J. 2019. Drop-in biofuel production using fatty acid photodecarboxylase from Chlorella variabilis in the oleaginous yeast Yarrowia lipolytica. Biotechnol. Biofuels 12(1), 202.

11. Cao, X., Wei, L.-J., Lin, J.-Y., Hua, Q. 2017. Enhancing linalool production by engineering oleaginous yeast Yarrowia lipolytica. Bioresour. Technol. 245, 16411644.

12. Carly, F., Steels, S., Telek, S., Vandermies, M., Nicaud, J.-M., Fickers, P. 2018. Identification and characterization of EYD1, encoding an erythritol dehydrogenase in Yarrowia lipolytica and its application to bioconvert erythritol into erythrulose. Bioresour. Technol. 247, 963-969.

13. Darvishi, F., Fathi, Z., Ariana, M., Moradi, H. 2017. Yarrowia lipolytica as a workhorse for biofuel production. Biochem. Eng. J. 127, 87-96.

14. Daskalaki, A., Perdikouli, N., Aggeli, D., Aggelis, G. 2019. Laboratory evolution strategies for improving lipid accumulation in Yarrowia lipolytica. Appl. Microbiol. 
Biotechnol. 103(20), 8585-8596.

15. Dobrowolski, A., Drzymała, K., Rzechonek, D.A., Mituła, P., Mirończuk, A.M. 2019. Lipid Production From Waste Materials in Seawater-Based Medium by the Yeast Yarrowia lipolytica. Front. Microbiol. 10, 547.

16. Dobrowolski, A., Mituła, P., Rymowicz, W., Mirończuk, A.M. 2016. Efficient conversion of crude glycerol from various industrial wastes into single cell oil by yeast Yarrowia lipolytica. Bioresour. Technol. 207, 237-243.

17. Dourou, M., Aggeli, D., Papanikolaou, S., Aggelis, G. 2018. Critical steps in carbon metabolism affecting lipid accumulation and their regulation in oleaginous microorganisms. Appl. Microbiol. Biotechnol. 102(6), 2509-2523.

18. Dulermo, R., Dulermo, T., Gamboa-Meléndez, H., Thevenieau, F., Nicaud, J.-M. 2015a. Role of Pex11p in Lipid Homeostasis in Yarrowia lipolytica. Eukaryot. Cell 14(5), 511-525.

19. Dulermo, R., Gamboa-Meléndez, H., Ledesma-Amaro, R., Thévenieau, F., Nicaud, J.-M. 2015b. Unraveling fatty acid transport and activation mechanisms in Yarrowia lipolytica. Biochim. Biophys. Acta Mol. Cell Biol. Lipids 1851(9), 12021217.

20. Dulermo, R., Gamboa-Meléndez, H., Ledesma-Amaro, R., Thevenieau, F., Nicaud, J.-M. 2016. Yarrowia lipolytica AAL genes are involved in peroxisomal fatty acid activation. Biochim. Biophys. Acta Mol. Cell Biol. Lipids 1861(7), 555-565.

21. Dulermo, T., Nicaud, J.M. 2011. Involvement of the G3P shuttle and beta-oxidation pathway in the control of TAG synthesis and lipid accumulation in Yarrowia lipolytica. Metab. Eng. 13(5), 482-91.

22. Dulermo, T., Treton, B., Beopoulos, A., Kabran Gnankon, A.P., Haddouche, R., Nicaud, J.M. 2013. Characterization of the two intracellular lipases of Y. lipolytica encoded by TGL3 and TGL4 genes: new insights into the role of intracellular lipases and lipid body organisation. Biochim. Biophys. Acta Mol. Cell Biol. Lipids 1831(9), 1486-95.

23. Evans, C.T., Scragg, A.H., Ratledge, C. 1983. Regulation of citrate efflux from mitochondria of oleaginous and non-oleaginous yeasts by long-chain fatty acylCoA esters. Eur. J. Biochem. 132(3), 617-622.

24. Flowers, M.T., Ntambi, J.M. 2008. Role of stearoyl-coenzyme A desaturase in regulating lipid metabolism. Curr Opin Lipidol. 19(3), 248-256.

25. Friedlander, J., Tsakraklides, V., Kamineni, A., Greenhagen, E.H., Consiglio, A.L., MacEwen, K., et al. 2016. Engineering of a high lipid producing Yarrowia lipolytica strain. Biotechnol. Biofuels 9, 77.

26. Fukuda, R. 2013. Metabolism of Hydrophobic Carbon Sources and Regulation of It in n-Alkane-Assimilating Yeast Yarrowia lipolytica. Biosci. Biotechnol. Biochem. 77(6), 1149-1154.

27. Gajdoš, P., Nicaud, J.-M., Čertík, M. 2017. Glycerol conversion into a single cell oil by engineered Yarrowia lipolytica. Eng. Life Sci. 17(3), 325-332.

28. Gajdos, P., Nicaud, J.M., Rossignol, T., Certik, M. 2015. Single cell oil production on molasses by Yarrowia lipolytica strains overexpressing DGA2 in multicopy. Appl. Microbiol. Biotechnol. 99(19), 8065-74. 
29. Gao, S., Tong, Y., Wen, Z., Zhu, L., Ge, M., Chen, D., et al. 2016. Multiplex gene editing of the Yarrowia lipolytica genome using the CRISPR-Cas9 system. J. Ind. Microbiol. Biotechnol. 43(8), 1085-1093.

30. Gemperlein, K., Dietrich, D., Kohlstedt, M., Zipf, G., Bernauer, H.S., Wittmann, C., et al. 2019. Polyunsaturated fatty acid production by Yarrowia lipolytica employing designed myxobacterial PUFA synthases. Nat. Commun. 10(1), 4055.

31. Grimsrud, P.A., Xie, H., Griffin, T.J., Bernlohr, D.A. 2008. Oxidative stress and covalent modification of protein with bioactive aldehydes. J. Biol. Chem. 283(32), 21837-21841.

32. Groenewald, M., Boekhout, T., Neuvéglise, C., Gaillardin, C., van Dijck, P.W.M., Wyss, M. 2014. Yarrowia lipolytica: Safety assessment of an oleaginous yeast with a great industrial potential. Crit. Rev. Microbiol. 40(3), 187-206.

33. Hapeta, P., Rakicka, M., Dulermo, R., Gamboa-Melendez, H., Cruz-Le Coq, A.M., Nicaud, J.M., et al. 2017. Transforming sugars into fat - lipid biosynthesis using different sugars in Yarrowia lipolytica. Yeast 34(7), 293-304.

34. Jacobsen, I.H., Ledesma-Amaro, R., Martinez, J.L. 2020. Recombinant $\beta$-Carotene Production by Yarrowia lipolytica - Assessing the Potential of Micro-Scale Fermentation Analysis in Cell Factory Design and Bioreaction Optimization. Front. Bioeng. Biotechnol. 8, 29.

35. Ji, X.-J., Huang, H. 2019. Engineering Microbes to Produce Polyunsaturated Fatty Acids. Trends Biotechnol. 37(4), 344-346.

36. Ji, X.-J., Ledesma-Amaro, R. 2020. Microbial Lipid Biotechnology to Produce Polyunsaturated Fatty Acids. Trends Biotechnol.

37. Johnravindar, D., Karthikeyan, O.P., Selvam, A., Murugesan, K., Wong, J.W.C. 2018. Lipid accumulation potential of oleaginous yeasts: A comparative evaluation using food waste leachate as a substrate. Bioresour. Technol. 248, 221-228.

38. Kamzolova, S.V., Morgunov, I.G. 2018. Biosynthesis of pyruvic acid from glycerolcontaining substrates and its regulation in the yeast Yarrowia lipolytica. Bioresour. Technol. 266, 125-133.

39. Kamzolova, S.V., Morgunov, I.G. 2017. Metabolic peculiarities of the citric acid overproduction from glucose in yeasts Yarrowia lipolytica. Bioresour. Technol. 243, 433-440.

40. Kavšček, M., Bhutada, G., Madl, T., Natter, K. 2015. Optimization of lipid production with a genome-scale model of Yarrowia lipolytica. BMC Syst. Biol. 9(1), 72 .

41. Kerkhoven, E.J., Pomraning, K.R., Baker, S.E., Nielsen, J. 2016. Regulation of amino-acid metabolism controls flux to lipid accumulation in Yarrowia lipolytica. npj. Syst. Biol. Appl. 2, 16005.

42. Kim, J.-Y., Lee, H.W., Lee, S.M., Jae, J., Park, Y.-K. 2019a. Overview of the recent advances in lignocellulose liquefaction for producing biofuels, bio-based materials and chemicals. Bioresour. Technol. 279, 373-384.

43. Kim, M., Park, B.G., Kim, E.J., Kim, J., Kim, B.G. 2019b. In silico identification of metabolic engineering strategies for improved lipid production in Yarrowia lipolytica by genome-scale metabolic modeling. Biotechnol. Biofuels 12, 187. 
44. Larroude, M., Celinska, E., Back, A., Thomas, S., Nicaud, J.-M., Ledesma-Amaro, R. 2018a. A synthetic biology approach to transform Yarrowia lipolytica into a competitive biotechnological producer of $\beta$-carotene. Biotechnol. Bioeng. 115(2), 464-472.

45. Larroude, M., Rossignol, T., Nicaud, J.M., Ledesma-Amaro, R. 2018b. Synthetic biology tools for engineering Yarrowia lipolytica. Biotechnol. Adv. 36(8), 21502164.

46. Lazar, Z., Dulermo, T., Neuvéglise, C., Crutz-Le Coq, A.-M., Nicaud, J.-M. 2014. Hexokinase-A limiting factor in lipid production from fructose in Yarrowia lipolytica. Metab. Eng. 26, 89-99.

47. Lazar, Z., Gamboa-Meléndez, H., Le Coq, A.-M.C., Neuvéglise, C., Nicaud, J.-M. 2015. Awakening the endogenous Leloir pathway for efficient galactose utilization by Yarrowia lipolytica. Biotechnol. Biofuels 8(1), 185.

48. Lazar, Z., Liu, N., Stephanopoulos, G. 2018. Holistic Approaches in Lipid Production by Yarrowia lipolytica. Trends Biotechnol. 36(11), 1157-1170.

49. Ledesma-Amaro, R. 2015. Microbial oils: A customizable feedstock through metabolic engineering. Eur. J. Lipid Sci. Technol. 117(2), 141-144.

50. Ledesma-Amaro, R., Dulermo, R., Niehus, X., Nicaud, J.M. 2016a. Combining metabolic engineering and process optimization to improve production and secretion of fatty acids. Metab. Eng. 38, 38-46.

51. Ledesma-Amaro, R., Dulermo, T., Nicaud, J.M. 2015. Engineering Yarrowia lipolytica to produce biodiesel from raw starch. Biotechnol. Biofuels 8(1), 148.

52. Ledesma-Amaro, R., Lazar, Z., Rakicka, M., Guo, Z., Fouchard, F., Coq, A.C., et al. 2016b. Metabolic engineering of Yarrowia lipolytica to produce chemicals and fuels from xylose. Metab. Eng. 38, 115-124.

53. Ledesma-Amaro, R., Nicaud, J.-M. 2016a. Metabolic Engineering for Expanding the Substrate Range of Yarrowia lipolytica. Trends Biotechnol. 34(10), 798-809.

54. Ledesma-Amaro, R., Nicaud, J.-M. 2016b. Yarrowia lipolytica as a biotechnological chassis to produce usual and unusual fatty acids. Prog. Lipid Res. 61, 40-50.

55. Lei, Q., Zeng, W., Zhou, J., Du, G. 2019. Efficient separation of $\alpha$-ketoglutarate from Yarrowia lipolytica WSH-Z06 culture broth by converting pyruvate to 1tyrosine. Bioresour. Technol. 292, 121897.

56. Leplat, C., Nicaud, J.-M., Rossignol, T. 2018. Overexpression screen reveals transcription factors involved in lipid accumulation in Yarrowia lipolytica. FEMS Yeast Res. 18(5).

57. Li, C., Gao, S., Yang, X., Lin, C.S.K. 2018. Green and sustainable succinic acid production from crude glycerol by engineered Yarrowia lipolytica via agricultural residue based in situ fibrous bed bioreactor. Bioresour. Technol. 249, 612-619.

58. Li, C., Yang, X., Gao, S., Wang, H., Lin, C.S.K. 2017. High efficiency succinic acid production from glycerol via in situ fibrous bed bioreactor with an engineered Yarrowia lipolytica. Bioresour. Technol. 225, 9-16.

59. Li, H., Alper, H.S. 2016. Enabling xylose utilization in Yarrowia lipolytica for lipid production. Biotechnol. J. 11(9), 1230-1240.

60. Li, Q., Bai, Z., O’Donnell, A., Harvey, L.M., Hoskisson, P.A., McNeil, B. 2011. 
Oxidative stress in fungal fermentation processes: the roles of alternative respiration. Biotechnol. Lett. 33(3), 457-467.

61. Liu, H.-H., Ji, X.-J., Huang, H. 2015a. Biotechnological applications of Yarrowia lipolytica: Past, present and future. Biotechnol. Adv. 33(8), 1522-1546.

62. Liu, H.-H., Madzak, C., Sun, M.-L., Ren, L.-J., Song, P., Huang, H., et al. 2017a. Engineering Yarrowia lipolytica for arachidonic acid production through rapid assembly of metabolic pathway. Biochem. Eng. J. 119, 52-58.

63. Liu, H.-H., Wang, C., Lu, X.-Y., Huang, H., Tian, Y., Ji, X.-J. 2019a. Improved Production of Arachidonic Acid by Combined Pathway Engineering and Synthetic Enzyme Fusion in Yarrowia lipolytica. J. Agric. Food Chem. 67(35), 9851-9857.

64. Liu, H.-H., Zeng, S.-Y., Shi, T.-Q., Ding, Y., Ren, L.-J., Song, P., et al. 2017b. A Yarrowia lipolytica strain engineered for arachidonic acid production counteracts metabolic burden by redirecting carbon flux towards intracellular fatty acid accumulation at the expense of organic acids secretion. Biochem. Eng. J. 128, 201209.

65. Liu, L., Alper, H.S. 2014. Draft Genome Sequence of the Oleaginous Yeast Yarrowia lipolytica PO1f, a Commonly Used Metabolic Engineering Host. Genome Announc. 2(4), e00652-14.

66. Liu, L., Pan, A., Spofford, C., Zhou, N., Alper, H.S. 2015b. An evolutionary metabolic engineering approach for enhancing lipogenesis in Yarrowia lipolytica. Metab. Eng. 29, 36-45.

67. Liu, L., Markham, K., Blazeck, J., Zhou, N., Leon, D., Otoupal, P., et al. 2015c. Surveying the lipogenesis landscape in Yarrowia lipolytica through understanding the function of a Mga2p regulatory protein mutant. Metab. Eng. 31, 102-11.

68. Liu, X., Yan, Y., Zhao, P., Song, J., Yu, X., Wang, Z., et al. 2019b. Oil crop wastes as substrate candidates for enhancing erythritol production by modified Yarrowia lipolytica via one-step solid state fermentation. Bioresour. Technol. 294, 122194.

69. Liu, X., Yu, X., Xia, J., Lv, J., Xu, J., Dai, B., et al. 2017c. Erythritol production by Yarrowia lipolytica from okara pretreated with the in-house enzyme pools of fungi. Bioresour. Technol. 244, 1089-1095.

70. Liu, Y., Jiang, X., Cui, Z., Wang, Z., Qi, Q., Hou, J. 2019c. Engineering the oleaginous yeast Yarrowia lipolytica for production of $\alpha$-farnesene. Biotechnol. Biofuels 12(1), 296.

71. Ma, Y.-R., Wang, K.-F., Wang, W.-J., Ding, Y., Shi, T.-Q., Huang, H., et al. 2019. Advances in the metabolic engineering of Yarrowia lipolytica for the production of terpenoids. Bioresour. Technol. 281, 449-456.

72. Ma, Y., Gao, Z., Wang, Q., Liu, Y. 2018. Biodiesels from microbial oils: Opportunity and challenges. Bioresour. Technol. 263, 631-641.

73. Magdouli, S., Guedri, T., Tarek, R., Brar, S.K., Blais, J.F. 2017. Valorization of raw glycerol and crustacean waste into value added products by Yarrowia lipolytica. Bioresour. Technol. 243, 57-68.

74. Mano, J.i., Torii, Y., Hayashi, S.-i., Takimoto, K., Matsui, K., Nakamura, K., et al. 2002. The NADPH:Quinone Oxidoreductase P1- $\zeta$-crystallin in Arabidopsis Catalyzes the $\alpha, \beta$-Hydrogenation of 2-Alkenals: Detoxication of the Lipid 
Peroxide-Derived Reactive Aldehydes. Plant Cell Physiol. 43(12), 1445-1455.

75. Mathiazhakan, K., Ayed, D., Tyagi, R.D. 2016. Kinetics of lipid production at lab scale fermenters by a new isolate of Yarrowia lipolytica SKY7. Bioresour. Technol. 221, 234-240.

76. Meullemiestre, A., Breil, C., Abert-Vian, M., Chemat, F. 2016. Microwave, ultrasound, thermal treatments, and bead milling as intensification techniques for extraction of lipids from oleaginous Yarrowia lipolytica yeast for a biojetfuel application. Bioresour. Technol. 211, 190-199.

77. Mlickova, K., Roux, E., Athenstaedt, K., d'Andrea, S., Daum, G., Chardot, T., et al. 2004. Lipid accumulation, lipid body formation, and acyl coenzyme A oxidases of the yeast Yarrowia lipolytica. Appl. Environ. Microbiol. 70(7), 3918-24.

78. Morin, N., Cescut, J., Beopoulos, A., Lelandais, G., Le Berre, V., Uribelarrea, J.L., et al. 2011. Transcriptomic analyses during the transition from biomass production to lipid accumulation in the oleaginous yeast Yarrowia lipolytica. PLoS One 6(11), e27966.

79. Nambou, K., Zhao, C., Wei, L., Chen, J., Imanaka, T., Hua, Q. 2014. Designing of a "cheap to run" fermentation platform for an enhanced production of single cell oil from Yarrowia lipolytica DSM3286 as a potential feedstock for biodiesel. Bioresour. Technol. 173, 324-333.

80. Niehus, X., Crutz-Le Coq, A.M., Sandoval, G., Nicaud, J.M., Ledesma-Amaro, R. 2018. Engineering Yarrowia lipolytica to enhance lipid production from lignocellulosic materials. Biotechnol. Biofuels 11, 11.

81. Palmieri, L., Palmieri, F., Runswick, M.J., Walker, J.E. 1996. Identification by bacterial expression and functional reconstitution of the yeast genomic sequence encoding the mitochondrial dicarboxylate carrier protein. FEBS Lett. 399(3), 299302.

82. Pan, P., Hua, Q. 2012. Reconstruction and In Silico Analysis of Metabolic Network for an Oleaginous Yeast, Yarrowia lipolytica. PLoS One 7(12), e51535.

83. Papanikolaou, S., Aggelis, G. 2011. Lipids of oleaginous yeasts. Part I: Biochemistry of single cell oil production. Eur. J. Lipid Sci. Technol. 113(8), 10311051.

84. Papanikolaou, S., Aggelis, G. 2003. Modeling Lipid Accumulation and Degradation in Yarrowia lipolytica Cultivated on Industrial Fats. Curr. Microbiol. 46(6), 03980402.

85. Park, Y.-K., Dulermo, T., Ledesma-Amaro, R., Nicaud, J.-M. 2018. Optimization of odd chain fatty acid production by Yarrowia lipolytica. Biotechnol. Biofuels 11(1), 158.

86. Park, Y.-k., Ledesma-Amaro, R., Nicaud, J.-M. 2020. De novo Biosynthesis of OddChain Fatty Acids in Yarrowia lipolytica Enabled by Modular Pathway Engineering. Front. Bioeng. Biotechnol. 7, 484.

87. Poli, J.S., da Silva, M.A.N., Siqueira, E.P., Pasa, V.M.D., Rosa, C.A., Valente, P. 2014. Microbial lipid produced by Yarrowia lipolytica QU21 using industrial waste: A potential feedstock for biodiesel production. Bioresour. Technol. 161, 320326. 
88. Qiao, K., Imam Abidi, S.H., Liu, H., Zhang, H., Chakraborty, S., Watson, N., et al. 2015. Engineering lipid overproduction in the oleaginous yeast Yarrowia lipolytica. Metab. Eng. 29, 56-65.

89. Qiao, K., Wasylenko, T.M., Zhou, K., Xu, P., Stephanopoulos, G. 2017. Lipid production in Yarrowia lipolytica is maximized by engineering cytosolic redox metabolism. Nat. Biotechnol. 35(2), 173-177.

90. Rakicka, M., Lazar, Z., Dulermo, T., Fickers, P., Nicaud, J.M. 2015. Lipid production by the oleaginous yeast Yarrowia lipolytica using industrial by-products under different culture conditions. Biotechnol. Biofuels 8(1), 104.

91. Rosenwasser, S., Graff van Creveld, S., Schatz, D., Malitsky, S., Tzfadia, O., Aharoni, A., et al. 2014. Mapping the diatom redox-sensitive proteome provides insight into response to nitrogen stress in the marine environment. Proc. Natl. Acad. Sci. U. S. A. 111(7), 2740-2745.

92. Rzechonek, D.A., Dobrowolski, A., Rymowicz, W., Mirończuk, A.M. 2019. Aseptic production of citric and isocitric acid from crude glycerol by genetically modified Yarrowia lipolytica. Bioresour. Technol. 271, 340-344.

93. Sagnak, R., Cochot, S., Molina-Jouve, C., Nicaud, J.M., Guillouet, S.E. 2018. Modulation of the Glycerol Phosphate availability led to concomitant reduction in the citric acid excretion and increase in lipid content and yield in Yarrowia lipolytica. J. Biotechnol. 265, 40-45.

94. Seip, J., Jackson, R., He, H., Zhu, Q., Hong, S.-P. 2013. Snf1 Is a Regulator of Lipid Accumulation in Yarrowia lipolytica. Appl. Environ. Microbiol. 79(23), 7360.

95. Sestric, R., Munch, G., Cicek, N., Sparling, R., Levin, D.B. 2014. Growth and neutral lipid synthesis by Yarrowia lipolytica on various carbon substrates under nutrient-sufficient and nutrient-limited conditions. Bioresour. Technol. 164, 41-46.

96. Shaw, A.J., Lam, F.H., Hamilton, M., Consiglio, A., MacEwen, K., Brevnova, E.E., et al. 2016. Metabolic engineering of microbial competitive advantage for industrial fermentation processes. Science 353(6299), 583.

97. Shi, K., Gao, Z., Shi, T.Q., Song, P., Ren, L.J., Huang, H., et al. 2017. Reactive Oxygen Species-Mediated Cellular Stress Response and Lipid Accumulation in Oleaginous Microorganisms: The State of the Art and Future Perspectives. Front. Microbiol. 8, 793.

98. Shi, T.-Q., Huang, H., Kerkhoven, E.J., Ji, X.-J. 2018. Advancing metabolic engineering of Yarrowia lipolytica using the CRISPR/Cas system. Appl. Microbiol. Biotechnol. 102(22), 9541-9548.

99. Silverman, A.M., Qiao, K., Xu, P., Stephanopoulos, G. 2016. Functional overexpression and characterization of lipogenesis-related genes in the oleaginous yeast Yarrowia lipolytica. Appl. Microbiol. Biotechnol. 100(8), 3781-3798.

100. Sun, M.-L., Madzak, C., Liu, H.-H., Song, P., Ren, L.-J., Huang, H., et al. 2017. Engineering Yarrowia lipolytica for efficient $\gamma$-linolenic acid production. Biochem. Eng. J. 117, 172-180.

101. Tai, M., Stephanopoulos, G. 2013. Engineering the push and pull of lipid biosynthesis in oleaginous yeast Yarrowia lipolytica for biofuel production. Metab. Eng. 15, 1-9. 
102. Trentacoste, E.M., Shrestha, R.P., Smith, S.R., Glé, C., Hartmann, A.C., Hildebrand, M., et al. 2013. Metabolic engineering of lipid catabolism increases microalgal lipid accumulation without compromising growth. Proc. Natl. Acad. Sci. U. S. A. 110(49), 19748.

103. Walker, C., Ryu, S., Giannone, R.J., Garcia, S., Trinh, C.T. 2020. Understanding and eliminating the detrimental effect of thiamine deficiency on the oleaginous yeast Yarrowia lipolytica. Appl. Environ. Microbiol. 86(3), e02299-19.

104. Wang, G., Li, D., Miao, Z., Zhang, S., Liang, W., Liu, L. 2018. Comparative transcriptome analysis reveals multiple functions for Mhy1p in lipid biosynthesis in the oleaginous yeast Yarrowia lipolytica. Biochim. Biophys. Acta Mol. Cell Biol. Lipids 1863(1), 81-90.

105. Wang, L., Zong, Z., Liu, Y., Zheng, M., Li, D., Wang, C., et al. 2019. Metabolic engineering of Yarrowia lipolytica for the biosynthesis of crotonic acid. Bioresour. Technol. 287, 121484.

106. Wang, Z.P., Xu, H.M., Wang, G.Y., Chi, Z., Chi, Z.M. 2013. Disruption of the MIG1 gene enhances lipid biosynthesis in the oleaginous yeast Yarrowia lipolytica ACA-DC 50109. Biochim. Biophys. Acta Mol. Cell Biol. Lipids 1831(4), 675-82.

107. Wasylenko, T.M., Ahn, W.S., Stephanopoulos, G. 2015. The oxidative pentose phosphate pathway is the primary source of NADPH for lipid overproduction from glucose in Yarrowia lipolytica. Metab. Eng. 30, 27-39.

108. Wei, S., Jian, X., Chen, J., Zhang, C., Hua, Q. 2017. Reconstruction of genomescale metabolic model of Yarrowia lipolytica and its application in overproduction of triacylglycerol. Bioresour. Bioprocess. 4(1), 51.

109. Xu, J., Liu, N., Qiao, K., Vogg, S., Stephanopoulos, G. 2017a. Application of metabolic controls for the maximization of lipid production in semicontinuous fermentation. Proc. Natl. Acad. Sci. U. S. A. 114(27), E5308-E5316.

110. Xu, P., Qiao, K., Ahn, W.S., Stephanopoulos, G. 2016. Engineering Yarrowia lipolytica as a platform for synthesis of drop-in transportation fuels and oleochemicals. Proc. Natl. Acad. Sci. U. S. A. 113(39), 10848-10853.

111. Xu, P., Qiao, K., Stephanopoulos, G. 2017b. Engineering oxidative stress defense pathways to build a robust lipid production platform in Yarrowia lipolytica. Biotechnol. Bioeng. 114(7), 1521-1530.

112. Xu, Y., Holic, R., Hua, Q. 2020. Comparison and Analysis of Published Genomescale Metabolic Models of Yarrowia lipolytica. Biotechnol. Bioprocess Eng. 25(1), 53-61.

113. Xue, Z., Sharpe, P.L., Hong, S.P., Yadav, N.S., Xie, D., Short, D.R., et al. 2013. Production of omega-3 eicosapentaenoic acid by metabolic engineering of Yarrowia lipolytica. Nat. Biotechnol. 31(8), 734-40.

114. Yan, F.X., Dong, G.R., Qiang, S., Niu, Y.J., Hu, C.Y., Meng, Y.H. 2020. Overexpression of $\triangle 12, \triangle 15$-Desaturases for Enhanced Lipids Synthesis in Yarrowia lipolytica. Front. Microbiol. 11, 289.

115. Yang, X., Nambou, K., Wei, L., Hua, Q. 2016. Heterologous production of $\alpha-$ farnesene in metabolically engineered strains of Yarrowia lipolytica. Bioresour. Technol. 216, 1040-1048. 
116. Yuzbasheva, E.Y., Mostova, E.B., Andreeva, N.I., Yuzbashev, T.V., Fedorov, A.S., Konova, I.A., et al. 2018. A metabolic engineering strategy for producing free fatty acids by the Yarrowia lipolytica yeast based on impairment of glycerol metabolism. Biotechnol. Bioeng. 115(2), 433-443.

117. Yuzbasheva, E.Y., Mostova, E.B., Andreeva, N.I., Yuzbashev, T.V., Laptev, I.A., Sobolevskaya, T.I., et al. 2017. Co-expression of glucose-6-phosphate dehydrogenase and acyl-CoA binding protein enhances lipid accumulation in the yeast Yarrowia lipolytica. New Biotech. 39, 18-21.

118. Zeng, S.-Y., Liu, H.-H., Shi, T.-Q., Song, P., Ren, L.-J., Huang, H., et al. 2018. Recent Advances in Metabolic Engineering of Yarrowia lipolytica for Lipid Overproduction. Eur. J. Lipid Sci. Technol. 120(3), 1700352.

119. Zeng, W., Zhang, H., Xu, S., Fang, F., Zhou, J. 2017. Biosynthesis of keto acids by fed-batch culture of Yarrowia lipolytica WSH-Z06. Bioresour. Technol. 243, 10371043.

120. Zhang, H., Kang, X., Xiao, N., Gao, M., Zhao, Y., Zhang, B., et al. 2019. Intracellular expression of Vitreoscilla haemoglobin improves lipid production in Yarrowia lipolytica. Lett. Appl. Microbiol. 68(3), 248-257.

121. Zhang, H., Zhang, L., Chen, H., Chen, Y.Q., Chen, W., Song, Y., et al. 2014. Enhanced lipid accumulation in the yeast Yarrowia lipolytica by over-expression of ATP:citrate lyase from Mus musculus. J. Biotechnol. 192, 78-84.

122. Zhang, H., Zhang, L., Chen, H., Chen, Y.Q., Ratledge, C., Song, Y., et al. 2013. Regulatory properties of malic enzyme in the oleaginous yeast, Yarrowia lipolytica, and its non-involvement in lipid accumulation. Biotechnol. Lett. 35(12), 20912098.

123. Zhang, P., Wang, Z.-P., Sheng, J., Zheng, Y., Ji, X.-F., Zhou, H.-X., et al. 2018. High and efficient isomaltulose production using an engineered Yarrowia lipolytica strain. Bioresour. Technol. 265, 577-580.

124. Zhang, S., He, Y., Sen, B., Wang, G. 2020. Reactive oxygen species and their applications toward enhanced lipid accumulation in oleaginous microorganisms. Bioresour. Technol. 307, 123234.

125. Zimniak, P. 2011. Relationship of electrophilic stress to aging. Free Radic. Biol. Med. 51(6), 1087-1105. 


\section{Tables}

Table 1 The primary enzymes involved in TAG metabolism in $Y$. lipolytica.

Table 2 Summary of metabolic strategies for increasing lipid accumulation in Y. lipolytica. $\Delta$ followed by lowercase letters represents gene knockouts. Gene overexpression is represented by all uppercase letters. Desaturase genes are expressed through the following notation: $\triangle \#$ D. " $\times \#$ " refers to gene copy number. Some genes are preceded by an uppercase letter and a lowercase letter, which refer to the source of the gene. For example, $S c S U C 2$ refers to the SUC2 gene from Saccharomyces cerevisiae. Specially, "hp4d" and "TEFin" refer to promoters, $f-\Delta 15 D$ means $\Delta 15 D$ from flax. 
Table 1 The primary enzymes involved in TAG metabolism in Y. lipolytica.

\begin{tabular}{|c|c|c|c|}
\hline Gene & EC number & YL name & Encoded enzyme \\
\hline$A C L 1$ & EC 2.3.3.8 & YALI0E34793g & ATP-citrate lyase, subunit 1 \\
\hline$A C L 2$ & EC 2.3.3.8 & YALI0D24431g & ATP-citrate lyase, subunit 2 \\
\hline$A C C 1$ & EC 6.4.1.2 & YALI0C $11407 \mathrm{~g}$ & Acetyl-CoA carboxylase \\
\hline GUT1 & EC 2.7.1.30 & YALI0F00484g & Glycerol kinase \\
\hline$G P D 1$ & EC 1.1.1.18 & YALI0B02948g & $\mathrm{NAD}^{+}$-dependent G3P dehydrogenase \\
\hline GUT2 & EC 1.1.5.3 & YALI0B $13970 \mathrm{~g}$ & $\mathrm{FAD}^{+}$-dependent G3P dehydrogenase \\
\hline$S C T 1$ & EC 2.3.1.15 & YALI0C00209g & Glycerol-3-phosphate acyltransferase \\
\hline SLC1 & EC 2.3.1.51 & YALI0E18964g & 1-acyl-sn-G3P acyltransferase \\
\hline$P A P$ & EC 3.1.3.4 & YALI0D27016g & Phosphatidic acid phosphatase \\
\hline$D G A 1$ & EC 2.3.1.20 & YALI0E32769g & Acyl-CoA diacylglycerol acyltransferase 1 \\
\hline$D G A 2$ & EC 2.3.1.20 & YALI0D07986g & Acyl-CoA diacylglycerol acyltransferase 2 \\
\hline LROI & EC 2.3.1.158 & YALI0E16797g & Phospholipid diacylglycerol acyltransferase \\
\hline$A R E 1$ & EC 2.3.1.26 & YALI0F06578g & Acyl-CoA sterol acyltransferase \\
\hline$T G L 3$ & EC 3.1.1.3 & YALI0D $17534 \mathrm{~g}$ & Triacylglycerol lipase 3 \\
\hline$T G L 4$ & EC 3.1.1.3 & YALI0F10010g & Triacylglycerol lipase 4 \\
\hline FAAl & EC 6.2.1.3 & YALI0D17864g & Long chain fatty acyl-CoA synthetase \\
\hline PXA1 & - & YALI0A06655g & Peroxisomal acyl-CoA transporter, subunit 1 \\
\hline$P X A 2$ & - & YALI0D04246g & Peroxisomal acyl-CoA transporter, subunit 2 \\
\hline$A A L 1$ & EC 6.2.1.12 & YALIOE11979g & Acyl/aryl-CoA ligase \\
\hline$A A L 2$ & EC 6.2.1.12 & YALI0A14234g & Acyl/aryl-CoA ligase \\
\hline$A A L 3$ & EC 6.2.1.12 & YALI0E05951g & Acyl/aryl-CoA ligase \\
\hline$A A L 4$ & EC 6.2.1.12 & YALI0E12419g & Acyl/aryl-CoA ligase \\
\hline$A A L 5$ & EC 6.2.1.12 & YALI0F06556g & Acyl/aryl-CoA ligase \\
\hline$A A L 6$ & EC 6.2.1.12 & YALI0C05885g & Acyl/aryl-CoA ligase \\
\hline$A A L 7$ & EC 6.2.1.12 & YALI0E20405g & Acyl/aryl-CoA ligase \\
\hline$A A L 8$ & EC 6.2.1.12 & YALI0B07755g & Acyl/aryl-CoA ligase \\
\hline$A A L 9$ & EC 6.2.1.12 & YALI0A15103g & Acyl/aryl-CoA ligase \\
\hline$A A L 10$ & EC 6.2.1.12 & YALI0D17314g & Acyl/aryl-CoA ligase \\
\hline POX1 & EC 1.3.3.6 & YALI0E32835g & Fatty-acyl-CoA oxidase \\
\hline$P O X 2$ & EC 1.3.3.6 & YALI0F10857g & Fatty-acyl-CoA oxidase \\
\hline$P O X 3$ & EC 1.3.3.6 & YALI0D24750g & Fatty-acyl-CoA oxidase \\
\hline POX4 & EC 1.3.3.6 & YALI0E27654g & Fatty-acyl-CoA oxidase \\
\hline POX5 & EC 1.3.3.6 & YALI0C $23859 \mathrm{~g}$ & Fatty-acyl-CoA oxidase \\
\hline POX6 & EC 1.3.3.6 & YALI0E06567g & Fatty-acyl-CoA oxidase \\
\hline$M F E 1$ & EC 4.2.1.119 & YALI0E15378g & Peroxisomal multifunctional enzyme type 1 \\
\hline POT1 & EC 2.3.1.16 & YALI0E $18568 \mathrm{~g}$ & 3-ketoacyl-CoA thiolase \\
\hline$P E X 3$ & - & YALI0F22539g & Peroxisomal biogenesis factor 3 \\
\hline PEX10 & - & YALI0C01023g & Peroxisomal biogenesis factor 10 \\
\hline PEX11 & - & YALI0C04092g & Peroxisomal membrane protein \\
\hline$M A E$ & EC 1.1.1.38 & YALI0E18634g & Mitochondrial malic enzyme \\
\hline
\end{tabular}

Note: All information listed above comes from GRYC (http://gryc.inra.fr) and NCBI. 
Table 2 Summary of metabolic strategies for increasing lipid accumulation in $Y$. lipolytica. $\Delta$ followed by lowercase letters represents gene knockouts. Gene overexpression is represented by all uppercase letters. Desaturase genes are expressed through the following notation: $\Delta \# \mathrm{D}$. " $\times$ \#" refers to gene copy number. Some genes are preceded by an uppercase letter and a lowercase letter, which refer to the source of the gene. For example, ScSUC2 refers to the SUC2 gene from Saccharomyces cerevisiae. Specially, "hp4d" and "TEFin" refer to promoters, $f$ $\triangle 15 D$ means $\triangle 15 D$ from flax.

\begin{tabular}{|c|c|c|c|c|c|c|c|c|c|}
\hline Product & Substrate & Scale & Parental strain & Genetic modifications & $\begin{array}{l}\text { Content } \\
(\%)\end{array}$ & $\begin{array}{l}\text { Titer } \\
(\mathrm{g} / \mathrm{L})\end{array}$ & $\begin{array}{l}\text { Yield } \\
(\mathrm{g} / \mathrm{g})\end{array}$ & $\begin{array}{l}\text { Productivity } \\
(\mathrm{g} /(\mathrm{L} \cdot \mathrm{h}))\end{array}$ & References \\
\hline TAG & Glucose & Flask & Po1d & $\Delta g u t 2, \Delta p o x 1-6, G P D 1$ & 70.0 & - & - & - & Dulermo and Nicaud (2011) \\
\hline TAG & Glucose & Flask & Pold & $\triangle \operatorname{pox} 1-6, G P D 1$ & 65.0 & - & - & - & Dulermo and Nicaud (2011) \\
\hline TAG & Glucose & Flask & Pold & $\Delta m f e 1, G P D 1$ & 54.0 & - & - & - & Dulermo and Nicaud (2011) \\
\hline FA & Glycerol & Flask & W29 & $\begin{array}{l}\Delta d g a 1, \Delta d g a 2, \Delta \text { lrol, } \Delta a r e 1, \\
D G A 2 \times 4\end{array}$ & 53.7 & 9.90 & - & - & Gajdoš et al. (2017) \\
\hline TAG & Glycerol & Flask & Pold & $\Delta g s y 1, \Delta \operatorname{tgl} 4, D G A 2, G P D 1$ & 52.4 & 2.62 & - & - & Bhutada et al. (2017) \\
\hline TAG & Sucrose & Flask & Po1d & $\begin{array}{l}\Delta d g a 1, \Delta d g a 2, \Delta l r o 1, \Delta a r e 1, \\
\Delta m f e 1, D G A 2 \times 3, \text { ScSUC } 2\end{array}$ & 49.0 & 6.70 & - & - & Gajdos et al. (2015) \\
\hline TAG & Glucose & Flask & ACA-DC 50109 & $\Delta m i g 1$ & 48.7 & 2.44 & - & - & Wang et al. (2013) \\
\hline TAG & Glucose & Flask & ACA-DC 50109 & $\Delta m h y l$ & 43.1 & - & - & - & Wang et al. (2018) \\
\hline FA & Glucose & Flask & ATCC 20362 & $\Delta s n f 1$ & 30.0 & 1.10 & - & - & Seip et al. (2013) \\
\hline TAG & Glucose & Flask & W29 & $\triangle \operatorname{pex} 10, Z W F 1, A C B P$ & 30.0 & - & - & - & Yuzbasheva et al. (2017) \\
\hline TAG & Glycerol & Flask & Po1h & $M m A C L$ & 23.1 & 1.70 & - & - & Zhang et al. (2014) \\
\hline TAG & $\begin{array}{l}\text { Seawater/ } \\
\text { glycerol }\end{array}$ & Flask & AJD & $D G A 1$ & 21.0 & - & 0.070 & - & Dobrowolski et al. (2019) \\
\hline TAG & Starch & Flask & Po1d & $\begin{array}{l}\text { GPD1, (riceAlphaAmylase, } \\
\text { Glucoamylase }) \times 2\end{array}$ & - & 2.84 & 0.13 & - & Ledesma-Amaro et al. (2015) \\
\hline FFA & Glycerol & Flask & W29 & $\Delta g p d 1, \Delta g u t 2, \Delta p e x 10, A C C 1$ & - & 2.03 & - & - & Yuzbasheva et al. (2018) \\
\hline TAG & Galactose & Bioreactor & Pold & $\begin{array}{l}\text { GAL1, GAL7, GAL10E, } \\
\text { GAL10M }\end{array}$ & 16.6 & 3.22 & 0.056 & - & Lazar et al. (2015) \\
\hline
\end{tabular}




\begin{tabular}{|c|c|c|c|c|c|c|c|c|c|}
\hline TAG & Sucrose & Bioreactor & Pold & $\begin{array}{l}\Delta \operatorname{tgl4}, \triangle p o x 1-6, D G A 2, \\
G P D 1, H X K 1, S c S U C 2\end{array}$ & 26.0 & 9.15 & 0.063 & - & Lazar et al. (2014) \\
\hline FFA & Glucose & Bioreactor & W29 & $\begin{array}{l}\Delta f a a 1, \Delta m f e 1, D G A 2, T L G 4, \\
\text { KlTGL3 }\end{array}$ & 120.4 & 10.40 & 0.200 & & Ledesma-Amaro et al. (2016a) \\
\hline TAG & Xylose & Bioreactor & E26 & $\begin{array}{l}(\Delta p e x 10, \Delta m f e 1, D G A 1 \\
L E U 2, U R A 3, \text { evolved }) \\
\text { SsXYL1, SsXYL2, starved }\end{array}$ & - & 15.00 & - & 0.190 & Li and Alper (2016) \\
\hline TAG & $\begin{array}{l}\text { Glucose/ } \\
\text { xylose/ } \\
\text { acetate }\end{array}$ & Bioreactor & Pold & $\begin{array}{l}\triangle p o x 1-6, \Delta \operatorname{tgl}, G D P 1, \\
D G A 2, X R, X D H, X K, \\
A n X P K A, A n A C K\end{array}$ & 67.0 & 16.50 & 0.344 & 1.850 & Niehus et al. (2018) \\
\hline TAG & Glucose & Bioreactor & Po1g & $h p 4 d-A C C 1, T E F i n-D G A 1$ & 61.7 & 17.60 & 0.195 & 0.143 & Tai and Stephanopoulos (2013) \\
\hline FAMEs & $\begin{array}{l}\text { Glucose/ } \\
\text { Phosphite }\end{array}$ & Bioreactor & NS18 & $D G A 1, D G A 2, P s P T X D$ & - & 23.0 & - & - & Shaw et al. (2016) \\
\hline TAG & Inulin & Bioreactor & Pold & $\begin{array}{l}\triangle p o x 1-6, \triangle \operatorname{tgl} 4, D G A 2, \\
\text { GPD1, HXK1, GAL1, GAL7, } \\
\text { GAL10E, GAL10M, ScSUC2, } \\
\text { kmINU1 }\end{array}$ & 43.0 & 23.82 & 0.160 & 0.158 & Hapeta et al. (2017) \\
\hline TAG & $\begin{array}{l}\text { Molasses/ } \\
\text { glycerol }\end{array}$ & Bioreactor & Pold & $\begin{array}{l}\Delta p o x 1-6, \Delta \operatorname{tgl} l, D G A 2, \\
G P D 1, H X K 1, S c S U C 2\end{array}$ & 31.0 & 24.20 & 0.100 & 0.430 & Rakicka et al. (2015) \\
\hline TAG & Glucose & Bioreactor & L36 & $D G A 1, L E U 2, U R A 3$ & - & 25.00 & 0.213 & 0.145 & Liu et al. (2015b) \\
\hline TAG & Glucose & Bioreactor & Polf & $\begin{array}{l}\triangle p e x 10, \Delta m f e 1, D G A 1, L E U 2, \\
U R A 3\end{array}$ & 71.0 & 25.30 & - & 0.210 & Blazeck et al. (2014) \\
\hline TAG & Glucose & Bioreactor & Polf & $\begin{array}{l}\Delta p e x 10, \Delta m f e 1, D G A 1, L E U 2, \\
U R A 3, \text { evolved }\end{array}$ & 78.0 & 39.10 & 0.244 & 0.560 & Liu et al. (2015c) \\
\hline TAG & Glucose & Bioreactor & Po1f & $\begin{array}{l}A C C 1, D G A 1, S C D, \Delta 12 D, f- \\
\Delta 15 D, \Delta \operatorname{pex} 10, \Delta m f e 1\end{array}$ & 77.8 & 50.00 & - & - & Yan et al. (2020) \\
\hline TAG & $\begin{array}{l}\text { Xylose/ } \\
\text { glycerol }\end{array}$ & Bioreactor & Pold & $\begin{array}{l}\Delta \text { pox } 1-6, \Delta \operatorname{tgl} l, G D P 1, \\
D G A 2, X K, S s X R, S s X D H\end{array}$ & 42.0 & 50.50 & 0.120 & - & Ledesma-Amaro et al. (2016b) \\
\hline
\end{tabular}




\begin{tabular}{|c|c|c|c|c|c|c|c|c|c|}
\hline TAG & Glucose & Bioreactor & Polg & $\begin{array}{l}\text { TEFin-ACC1, TEFin-DGA1, } \\
\text { TEFin-SCD }\end{array}$ & 67.0 & 55.00 & 0.234 & 0.707 & Qiao et al. (2015) \\
\hline TAG & Glucose & Bioreactor & Po1g & $A C C 1, D G A 1, S c C A T 2$ & 70.0 & 66.40 & 0.229 & 0.565 & Xu et al. (2016) \\
\hline TAG & Glucose & Bioreactor & Polg & $\begin{array}{l}\text { ACCl, DGA1, URA3, } \\
\text { EcALDH, ScZWF, GSR, GPO }\end{array}$ & 81.4 & 72.70 & 0.252 & 0.970 & Xu et al. (2017b) \\
\hline TAG & Glucose & Bioreactor & NS18 & RtDGA1, CpDGA2, $\triangle \operatorname{tgl} 3$ & 77.0 & 85.00 & 0.210 & 0.730 & Friedlander et al. (2016) \\
\hline FAMEs & Glucose & Bioreactor & Polg & $\begin{array}{l}\text { ACC1, DGA1, McMCE2, } \\
\text { CaGAPC }\end{array}$ & 66.7 & 99.30 & 0.279 & 1.200 & Qiao et al. (2017) \\
\hline TAG & Acetic acid & Bioreactor & Polg & $A C C 1, D G A 1$ & 59.0 & 115.00 & 0.160 & 0.800 & Xu et al. (2017a) \\
\hline
\end{tabular}

Notes: Lipid content (\%), grams of lipid per gram CDW; Lipid titer (g/L), grams of lipid per liter of culture; Lipid yield (g/g), grams of lipid per gram of carbon substrate consumed; Lipid productivity $(\mathrm{g} /(\mathrm{L} \cdot \mathrm{h}))$, grams per lipid per liter of culture per hour. 


\section{Figures}

Figure 1 Synthesis and degradation of TAGs in Y. lipolytica. Differently colored arrows indicate different metabolic pathways: black, glycolysis; green, oxidative pentose phosphate pathway; light blue, tricarboxylic acid (TCA) cycle and related anaplerotic reactions; yellow, de novo TAG synthesis pathway; purple, ex novo TAG synthesis pathway; navy blue, Kennedy pathway; red, $\beta$-oxidation pathway. Abbreviations: MIT, mitochondria; N, nucleus; ER, endoplasmic reticulum; LB, lipid body; PER, peroxisome; OAA, oxaloacetate; DHAP, dihydroxyacetone phosphate; G3P, glycerol-3-phosphate; LPA, lysophosphatidic acid; PA, phosphatidic acid; DAG, diacylglycerol; TAG, triacylglyceride; FFA, free fatty acid.

Figure 2. Overview of the different approaches for increasing lipid accumulation in $Y$. lipolytica. Abbreviations: TAG, triacylglyceride; $\Delta$, gene knockout; TEF, gene overexpression; ROS, reactive oxygen species; DAG, diacylglycerol; FFA, free fatty acid; $\beta, \beta$-oxidation pathway. (I) $\beta$-oxidation pathway is deleted to hinder lipid degradation; Regulating endogenous genes involved in lipid metabolism to push and pull lipid synthesis; Overexpressing heterologous genes to enhance enzyme activity or change cell physiology; Blocking other competing metabolic pathways to channel the metabolic flux towards lipid synthesis. (II) Engineering cytosolic NADPH pathways to provide more cofactors for lipogenesis; Engineering alternative acetyl-CoA pathways to decouple nitrogen limitation and provide more precursors for lipogenesis; Engineering oxidative stress defense pathways to mitigate lipotoxicity caused by ROS. (III) Engineering fatty acid secretion pathways to exceed the lipid storage limit of individual cells and facilitate product extraction. (IV) Growth-related adaptive laboratory evolution combined with metabolic engineering can maximize the robustness and productivity of engineered strain. (V) Expanding the range of available substrates to minimize production costs. (VI) Multiomics analysis to understand the regulatory mechanisms and bottlenecks in lipid metabolism, in silico metabolic models to design and optimize more efficient synthetic pathways, and synthetic biology tools to assemble and fine-tune Y. lipolytica metabolism. 


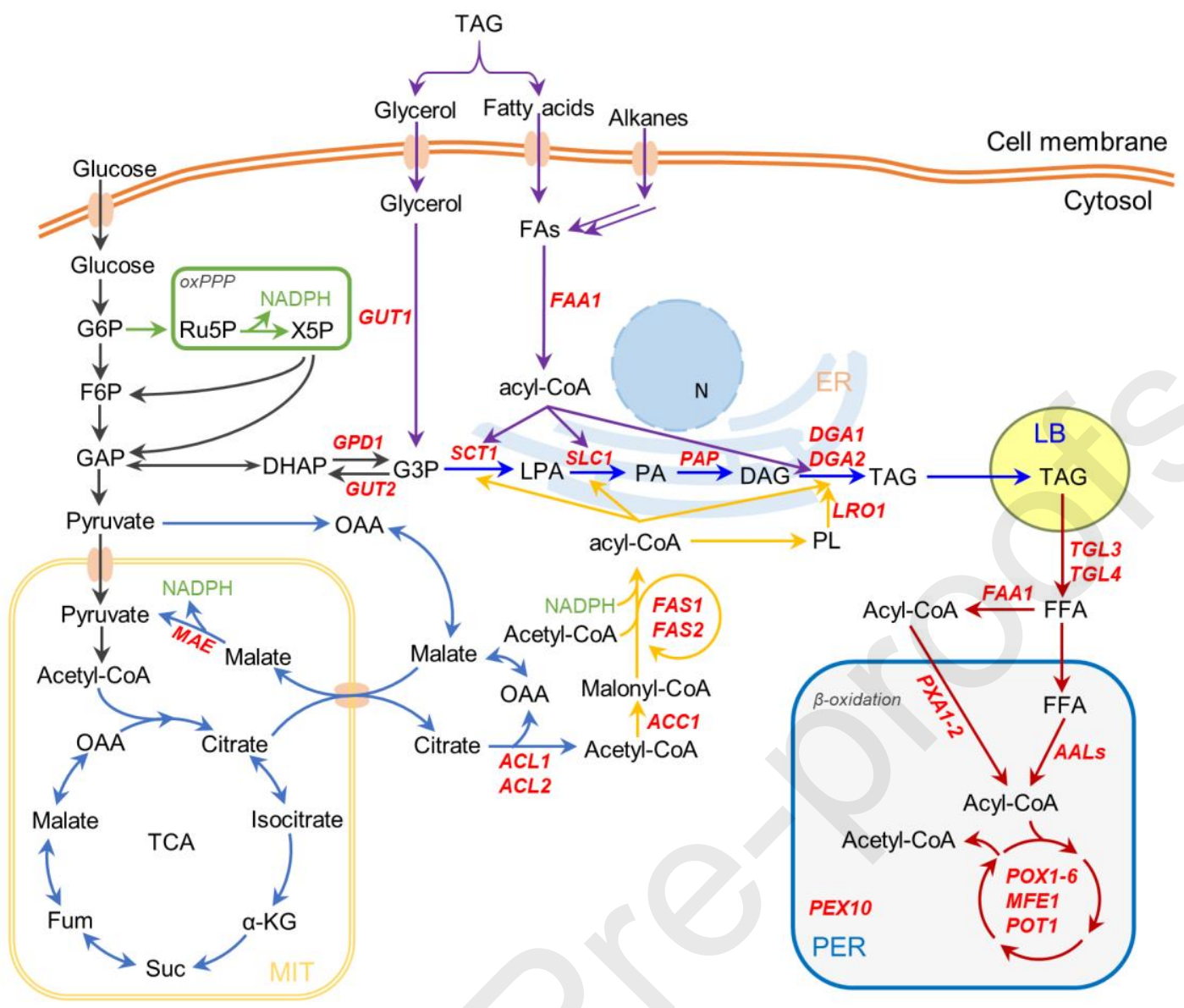

Figure 1 Synthesis and degradation of TAGs in Y. lipolytica. Differently colored arrows indicate different metabolic pathways: black, glycolysis; green, oxidative pentose phosphate pathway; light blue, tricarboxylic acid (TCA) cycle and related anaplerotic reactions; yellow, de novo TAG synthesis pathway; purple, ex novo TAG synthesis pathway; navy blue, Kennedy pathway; red, $\beta$-oxidation pathway. Abbreviations: MIT, mitochondria; N, nucleus; ER, endoplasmic reticulum; LB, lipid body; PER, peroxisome; OAA, oxaloacetate; DHAP, dihydroxyacetone phosphate; G3P, glycerol-3phosphate; LPA, lysophosphatidic acid; PA, phosphatidic acid; DAG, diacylglycerol; TAG, triacylglyceride; FFA, free fatty acid. 


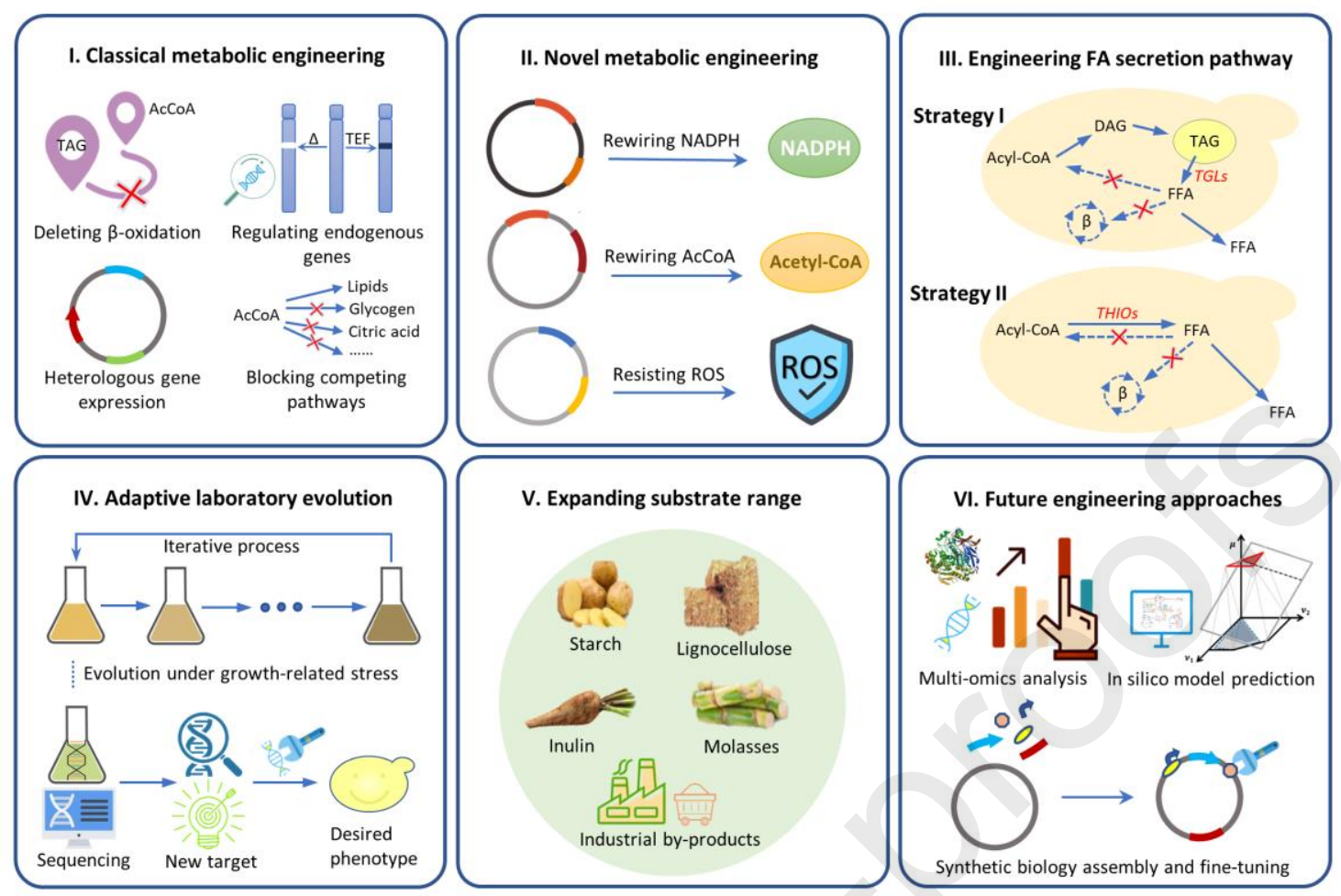

Figure 2. Overview of the different approaches for increasing lipid accumulation in Y. lipolytica. Abbreviations: TAG, triacylglyceride; $\Delta$, gene knockout; TEF, gene overexpression; ROS, reactive oxygen species; DAG, diacylglycerol; FFA, free fatty acid; $\beta$, $\beta$-oxidation pathway. (I) $\beta$-oxidation pathway is deleted to hinder lipid degradation; Regulating endogenous genes involved in lipid metabolism to push and pull lipid synthesis; Overexpressing heterologous genes to enhance enzyme activity or change cell physiology; Blocking other competing metabolic pathways to channel the metabolic flux towards lipid synthesis. (II) Engineering cytosolic NADPH pathways to provide more cofactors for lipogenesis; Engineering alternative acetyl-CoA pathways to decouple nitrogen limitation and provide more precursors for lipogenesis; Engineering oxidative stress defense pathways to mitigate lipotoxicity caused by ROS. (III) Engineering fatty acid secretion pathways to exceed the lipid storage limit of individual cells and facilitate product extraction. (IV) Growth-related adaptive laboratory evolution combined with metabolic engineering can maximize the robustness and productivity of engineered strain. (V) Expanding the range of available substrates to minimize production costs. (VI) Multi-omics analysis to understand the regulatory mechanisms and bottlenecks in lipid metabolism, in silico metabolic models to design and optimize more efficient synthetic pathways, and synthetic biology tools to assemble and fine-tune Y. lipolytica metabolism. 


\section{Graphic Abstract}

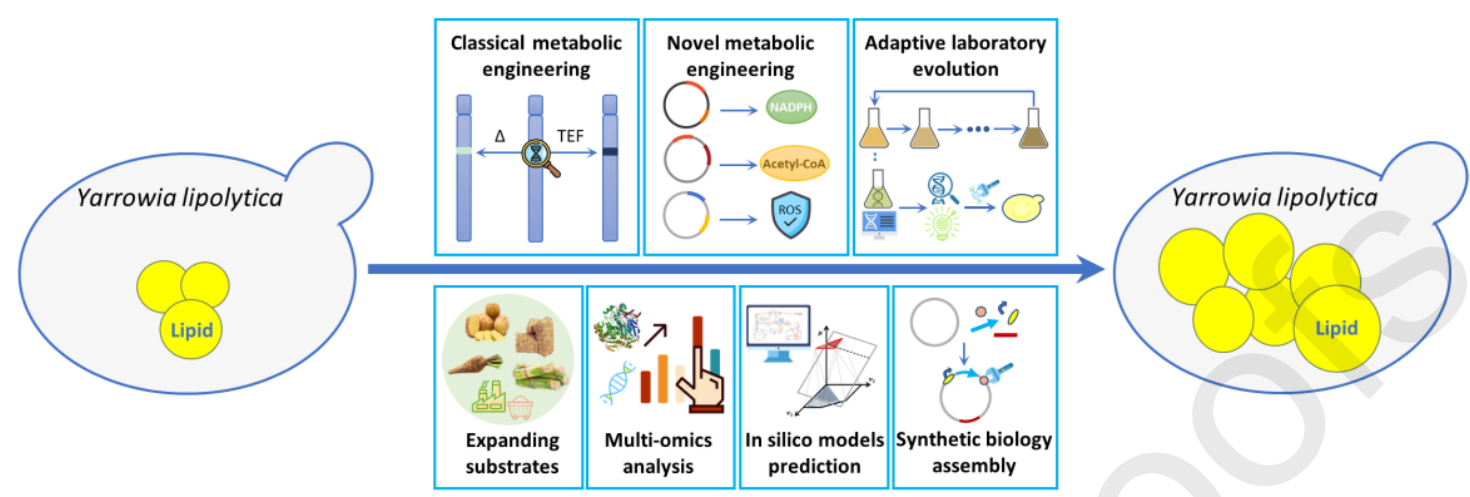

- $\quad$. lipolytica is a superior industrial host for the production of lipids.

- Biosynthesis and degradation pathways of lipid in Y. lipolytica and the potential metabolic engineering targets are introduced.

- Metabolic engineering strategies for increasing lipid accumulation in Y. lipolytica are summarized.

- Multi-omics analysis, in silico metabolic models, and synthetic biology tools will further push the lipid yields to theoretical limits.

- Perspectives for novel engineering approaches for increasing lipid accumulation in $Y$. lipolytica are discussed. 

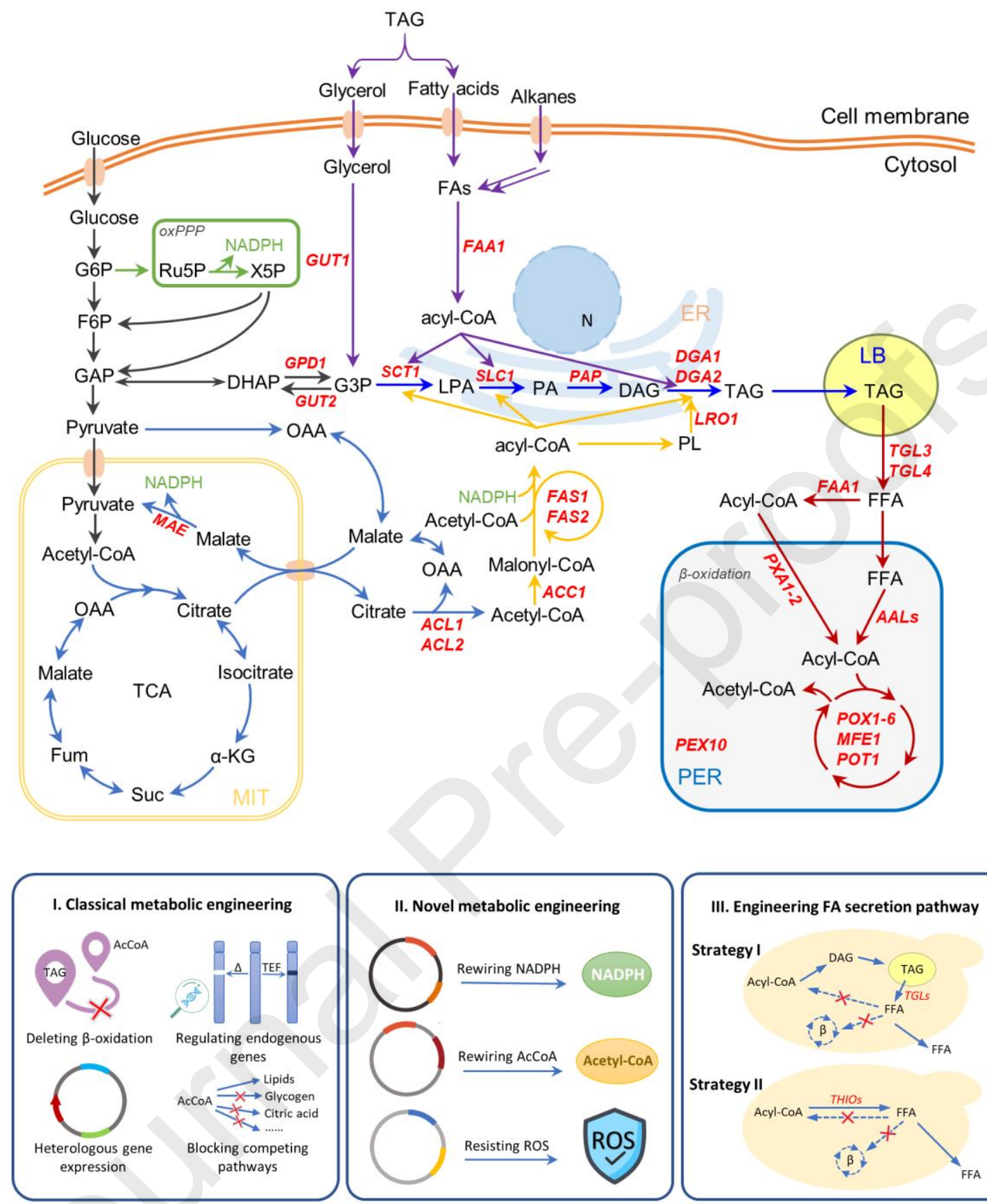

III. Engineering FA secretion pathway

Strategy I

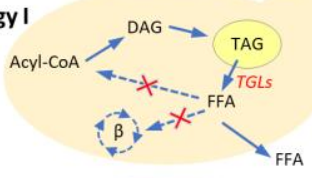

Strategy II

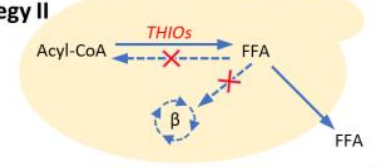

IV. Adaptive laboratory evolution

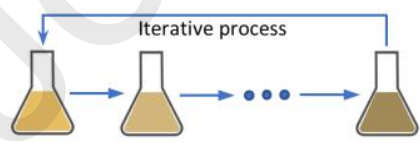

Evolution under growth-related stress

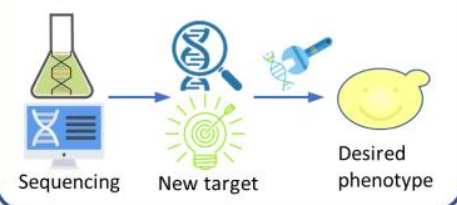

V. Expanding substrate range

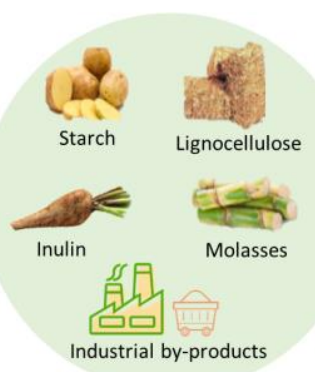

VI. Future engineering approaches 然 Multi-omics analysis in silico model prediction

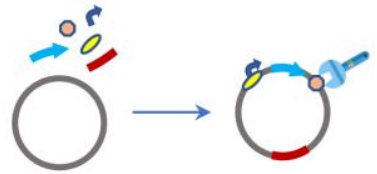




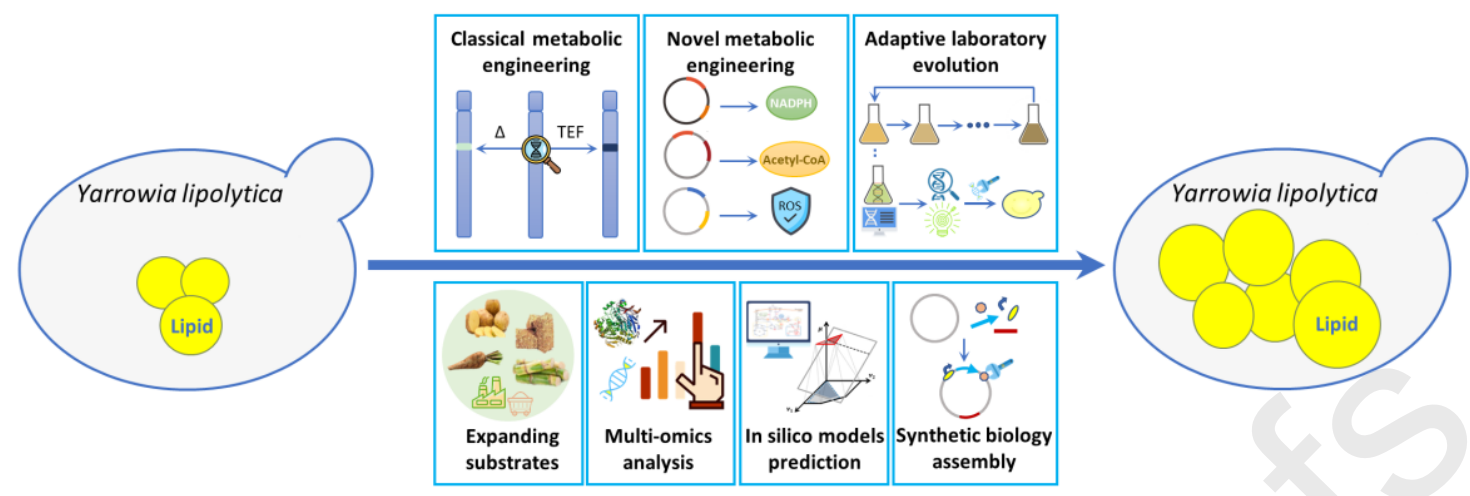

\section{Declaration of interest}

We declare that we have no financial and personal relationships with other people or organizations that can inappropriately influence our work, there is no professional or other personal interest of any nature or kind in any product, service and/or company that could be construed as influencing the position presented in, or the review of, the manuscript entitled, "Metabolic engineering for increased lipid accumulation in Yarrowia lipolytica". 